\title{
Adult North Star Network (ANSN): Consensus Guideline For The Standard Of Care Of Adults With Duchenne Muscular Dystrophy
}

R. Quinlivan ${ }^{\mathrm{a}, *}$, B. Messer ${ }^{\mathrm{b}}$, P. Murphy ${ }^{\mathrm{c}}$, R. Astin ${ }^{\mathrm{a}}$, R. Mukherjee ${ }^{\mathrm{d}}$, J. Khan ${ }^{\mathrm{a}}$, A. Emmanuel ${ }^{\mathrm{a}}$, S.C. Wong ${ }^{\mathrm{e}}$, R. Kulshresha ${ }^{\mathrm{f}}$, T. Willis ${ }^{\mathrm{f}}$, J. Pattni ${ }^{\mathrm{a}}$, D. Willis ${ }^{\mathrm{g}}$, A. Morgan ${ }^{\mathrm{h}}$, K. Savvatis $^{\mathrm{a}, \mathrm{i}}$, R. Keen ${ }^{\mathrm{j}}$, J. Bourke ${ }^{\mathrm{b}}$, C. Marini Bettolo ${ }^{\mathrm{b}}$ and C. Hewamadduma ${ }^{\mathrm{k}}$ on behalf of the ANSN

${ }^{a}$ MRC Centre for Neuromuscular Disease, UCL Institute of Neurology, National Hospital for Neurology and Neurosurgery, London, $U K$

${ }^{\mathrm{b}}$ Newcastle-upon-Tyne Hospitals NHS Foundation Trust, Newcastle, UK

'Lane Fox Unit, Guy's and St Thomas' Foundation Trust, London, UK

${ }^{\mathrm{d}}$ Heart of England NHS Foundation Trust, Birmingham, UK

${ }^{\mathrm{e}}$ University of Glasgow, Royal Hospital for Children, Glasgow, UK

${ }^{\mathrm{f}}$ Robert Jones and Agnes Hunt Foundation NHS Trust, Oswestry, UK

${ }^{\mathrm{g}}$ Shrewsbury and Telford NHS Trust, Shropshire, UK

${ }^{\mathrm{h}}$ South West Neuromuscular Operational Delivery Network, Bristol, UK

${ }^{i}$ St Bartholomew's Hospital and Royal London NHS Trust, London UK

${ }^{\mathrm{j}}$ Royal National Orthopaedic Hospital, Stanmore, UK

${ }^{\mathrm{k}}$ Academic Neurology Department, Sheffield Teaching Hospitals Foundation Trust and Sheffield Institute for Translational Neurosciences (SITRAN), University of Sheffield, Sheffield, UK

Pre-press 7 September 2021

\begin{abstract}
There are growing numbers of adults with Duchenne Muscular Dystrophy living well into their fourth decade. These patients have complex medical needs that to date have not been addressed in the International standards of care. We sought to create a consensus based standard of care through a series of multi-disciplinary workshops with specialists from a wide range of clinical areas: Neurology, Cardiology, Respiratory Medicine, Gastroenterology, Endocrinology, Palliative Care Medicine, Rehabilitation, Renal, Anaesthetics and Clinical Psychology. Detailed reports of evidence reviewed and the consensus building process were produced following each workshop and condensed into this final document which was approved by all members of the Adult North Star Network including service users. The aim of this document is to provide a framework to improve clinical services and multi-disciplinary care for adults living with Duchenne Muscular Dystrophy.
\end{abstract}

Keywords: Duchenne Muscular Dystrophy, non-invasive ventilation, multi-disciplinary care

\section{BACKGROUND}

${ }^{*}$ Correspondence to: R. Quinlivan, MRC Centre for Neuromuscular disease, UCL Institute of Neurology, National Hospital for Neurology and Neurosurgery, Queen Square, London, WC1 3BG, UK. E-mail: r.quinlivan@ucl.ac.uk.
People with Duchenne Muscular Dystrophy (DMD) are living longer due to improvements in the standard of care (SOC) [1-3]. The aim of this consensus building exercise is to add to, but not replace 
these standards of care, by placing more emphasis on the needs of the non-ambulant adult patient with more advanced disease. Most adults with DMD are frail and highly vulnerable, as their condition progresses they experience increasingly complex health issues including cardiac failure, cachexia, pain, renal dysfunction and bowel dysmotility that do not generally occur in childhood. Thus, for adult patients the emphasis of care shifts from a preventative approach to a treatment approach for example, drug treatment targeting the heart in adults with severe dilated cardiomyopathy treatment requires careful titration to avoid renal failure and there is additional risk of dysrhythmia and cerebrovascular embolism. Adults with DMD may require procedures such as percutaneous gastrostomy under general anaesthesia which, given their frailty, poses significant risk, this document provides more detailed specific guidance on general anaesthesia.

Most adult services have little experience in managing the complex needs of the adult with DMD and quite often multi-disciplinary care is fragmented with poor communication between specialists. The adult DMD patient community, and the clinicians caring for them in the UK, report variable levels of care accessible throughout the UK and a 'postcode lottery' in terms of quality of care. Consensus based care recommendations and emphasis on standards of care can reduce the inconsistencies in care provision across the country and thus influence clinical trial success in future for this patient population. The 'Adult North Star Network' (ANSN) was established in 2015 to improve care of adults with DMD living in the UK and to develop a prospective natural history database. The network comprises 28 adult centres (including two managed networks) caring for at least 700 DMD patients.

Complications related to DMD affect multiple systems and there is a paucity of high quality published evidence for managing such complex patients. In such circumstances consensus based management recommendations can standardize and improve the quality of care. Hopefully such gaps in evidence will be addressed in the future with large scale natural history studies.

\section{METHODOLOGY}

The ANSN network of clinicians and allied health professionals with experience in caring for adult DMD patients (from subspeciality areas including: neurology, rehabilitation medicine, clinical genetics, respiratory medicine, anaesthesiology, cardiology, gastroenterology, nephrology, endocrinology, palliative care medicine, psychology, physiotherapy, dietetics, speech and language therapy and occupational therapy), service users and representatives from patient advocacy groups participated in meetings in 2015, 2016 and 2017 to identify the need to modify the international SOC for adults with DMD in line with concerns raised about their care, a summary of the consensus building process is shown in Fig. 1. The ANSN steering group (comprising all 124 ANSN members) was established and a series of working subgroups identified. Expert workshops consisting of a variety of stakeholders (ASAN members including national experts in the various subspecialties and patient representatives/advocacy groups) were held by the whole network and then smaller working subgroups continued the consensus building process by reviewing the literature and current practices in more detail. Key management topics reviewed included: physiotherapy and musculoskeletal medicine, respiratory and general anaesthesia, cardiology, renal, nutrition and bowel, psychosocial and palliative care. Once the current evidence was evaluated, and where published evidence was lacking, consensus based opinion of best practice of care was made. This was achieved through reaching unanimous agreement based upon discussions and evaluations of the emerging themes reviewed by the expert groups. The outputs of these smaller subgroup workshops were summarised in individual reports, which were written by the workshop chair but then peer reviewed by workshop members, and these reports were subsequently presented at larger workshops encompassing the whole network where further discussion took place and unanimous consensus reached. Where there was a lack of good quality evidence in management for example gastroenterology and renal aspects of DMD care, we sought help from exisisting experts with experience in managing such issues in DMD adults, together with them we reviewed the literature and presented findings to the whole network for discussion and consensus on best management. Each of the subspecialty reports was then condensed into a single document which was circulated to all members of the ANSN for review and inviting discussion and comments via email together with an opportunity to discuss the final document face to face at a network meeting. Unanimous agreement for the final consensus document was reached by all parties without any areas of disagreement. In addition, all 
Stage 1

Email invitation to all UK physicians involved in adult DMD care

(from all relevant medical specialties), allied health care specialists and user groups

to join ANSN and form ANSN steering committee

Stage 2

Inaugural whole steering group workshop, action plan agreed on developing a consensus approach to care

Stage 3

Service user workshop held to understand unmet needs and health care priorities of adults living with DMD

Stage 4

Working subgroups (with neurologists caring for adults with DMD and national subspecialty experts with experience in the management of DMD) were identified in key areas: Respiratory, Anaesthesia, Cardiology, Renal, Psychology, Endocrinology and Palliative care. Leads for each area identified. Workshops held to discuss available published evidence and clinical experience. Broad discussion and comments were openly invited. There were no disagreements.

Stage 5

Chairs of each workshop completed a summary report in conjunction with individual workshop members. Each report was discussed at a ASAN whole group meeting (arranged specifically for each area topic) and the management plan for each topic presented and discussed with the whole group. There were no disagreements.

Stage 6

Each summary report was collated into a single document and sent to all 124 ASAN members (including patient representatives and advocacy groups) for peer review and invited to comment. All comments were collated and the document revised and emailed to all network members for final approval. There was unanimous approval ( $100 \%$ of network).

This final document was emailed to members for approval or rejection. There was unanimous approval ( $100 \%$ of network).

Fig. 1. Consensus building process. 
ASAN members were invited by email to comment on and accept or reject this final published manuscript (including any edits following journal peer review) the final published manuscript achieved unanimous $(100 \%)$ acceptance.

During the consensus building process, where possible, we used and adapted other evidence and consensus based guidelines including: the international standards of care for DMD [1-3], the most recent European Neuromuscular Centre (ENMC) workshop reports on bone protection [4] and cardiology care [5]. Steroid emergency guidance has been adapted from the recently published UK Endocrinology Society's recommendations [6] a link to which can also be found on their website (endocrinology.org). Guidelines for therapists participating in the DMD multi-disciplinary team (including physiotherapy, occupational therapy, speech and language therapy and dietetics) will be published in a separate document along with a therapy manual as supplementary material. For this reason, musculoskeletal care and scoliosis management are not included in this document. Transition care is an important topic and is mentioned here, although will be covered in more detail by the Paediatric North Star Network.

\section{RESPIRATORY}

\section{Introduction}

Respiratory failure is an inevitable consequence of the progression of DMD. In corticosteroid treated children, respiratory function improves, stabilizes and then declines at a rate of approximately 4-6.9\% per year from 9 years of age $[7,8]$. The onset of respiratory decline is delayed by treatment with corticosteroids, on average, by two years [7]. In adult services, only a minority of patients will require respiratory support at the time of transition from paediatric services, however, inevitably all patients will develop respiratory failure requiring ventilatory support, thereafter the trajectory of decline is the same, between $4-6.9 \%$ per year [7, 8]. Thus, services caring for adults with DMD must have access to a suitably skilled respiratory team, and either joint clinics or a clear referral pathway to a complex ventilation service should be in place. The objective is to anticipate deterioration to prevent, as far as possible, acute presentation to hospital, improve quality of life and prolong survival.

\section{Causes of respiratory pathology in DMD}

\section{Inspiratory muscle weakness}

Both obligate and accessory respiratory muscles weaken as the condition progresses, resulting in reduced alveolar ventilation. The involvement of the accessory muscles, such as intercostal and abdominal muscles, means there is little compensation for the impaired diaphragm as might occur in other conditions. During sleep and especially Rapid Eye Movement (REM) sleep, humans become obligate diaphragmatic breathers. Diaphragmatic weakness is therefore initially manifest overnight. This leads to nocturnal desaturation and hypercapnia causing arousal from sleep, fragmented sleep and symptoms which may include: disturbed sleep pattern, daytime sleepiness and early morning headaches. As weakness progresses, hypoventilation is associated with recurrent chest infections, inability to cough and swallowing impairment, whilst daytime hypercapnia leads to loss of appetite and some cognitive issues such as reduced attention, executive function and verbal delayed memory recall. However, these are late symptoms which may develop insidiously and are often not volunteered by patients. Adults with DMD need regular surveillance by clinicians who have experience in identifying the symptoms that may indicate the onset of nocturnal respiratory failure.

\section{Obstructive sleep apnoea}

Corticosteroid treated patients, who are Cushingoid, are also at risk of obstructive sleep apnoea (OSA). This may present with symptoms such as nocturnal snoring and/or daytime sleepiness which may warrant treatment [7]. Patients with isolated symptoms of OSA should be assessed by a respiratory/home ventilation team with experience in DMD management for further investigation such as overnight oximetry or polygraphy.

\section{Atelectasis, expiratory muscle weakness and aspiration}

A reduction in vital capacity results in areas of atelectasis increasing the risk of recurrent pulmonary infection. This is compounded by impaired clearance of respiratory secretions through reduced ability to cough adequately, due to a combination of inspiratory and expiratory muscle pathology with weakness affecting the diaphragm, intercostal and abdominal muscles. Poor swallow may increase the risk of aspiration and consequent pulmonary infection. 


\section{Measurement of respiratory function}

\section{Pulmonary function tests}

Respiratory failure is unlikely in ambulant patients and in non-ambulant patients when Forced vital Capacity $(\mathrm{FVC})>50 \%$ predicted. The risk of requiring ventilatory support increases as $\mathrm{FVC}$ falls below $50 \%$ and is very high when FVC falls below $30 \%$ [7, $8]$. Routine monitoring should include 6-12 monthly measurement of forced vital capacity (FVC) and peak cough flow (PCF), which is normally $>4001 / \mathrm{min}$. Maximum inspiratory and maximum expiratory mouth pressures are useful in the assessment of inspiratory and expiratory muscle weakness but have not been widely investigated in DMD. Referral to a specialist home ventilation clinic is recommended even in asymptomatic patients whose $\mathrm{FVC}$ is $<50 \%$ predicted. Referral should be made regardless of FVC in the presence of symptoms of either recurrent chest infection or nocturnal hypoventilation (morning headaches, fatigue, weight loss, poor sleep quality).

\section{Oxygen saturations}

Resting oxygen saturations cannot be relied upon to confirm or rule out respiratory failure. However, oxygen saturations $>95 \%$ makes significant hypercapnia unlikely $[7,9]$.

\section{Blood gases}

Raised daytime capillary or arterial partial pressures of carbon dioxide $\left(\mathrm{pCO}_{2}\right)$, or venous bicarbonate indicates the presence of advanced respiratory failure and require urgent referral to respiratory services. An elevated venous standard bicarbonate level (in the absence of other causes) indicates hypoventilation and should trigger further assessment.

\section{Overnight monitoring}

Overnight oximetry in the home can be useful for screening purposes; the presence of either intermittent or prolonged desaturation below $90 \%$ should warrant referral for a more detailed assessment by a respiratory specialist. Further investigations may include nocturnal oximetry and transcutaneous $\mathrm{CO}_{2}\left(\mathrm{TcCO}_{2}\right)$ monitoring, diurnal capillary or arterial blood gas analysis and standard bicarbonate measurement. The use of combined oximetry and $\mathrm{TcCO}_{2}$ monitoring will improve detection of nocturnal hypoventilation [9]

\section{Initiating non-invasive ventilation}

Guidelines for the management of neuromuscular weakness in children have been produced by the British Thoracic Society [10] and there is some published international guidance for adults with neuromuscular weakness [10, 11]. Respiratory management of adults with DMD should be led by a multi-disciplinary team familiar with the appropriate investigation, treatment and long term follow up of DMD patients which must be experienced and skilled in discussing the options for support with patients, as the literature is still inconclusive in defining which physiological tests or parameters mandate initiating ventilatory support. Clinical experience indicates that treatment is unlikely to be tolerated if the patient does not experience an increase in overall wellbeing. Thus, a shared decision-making framework is important because respiratory support via mask or tracheostomy and mechanical insufflation/exsufflation may be burdensome and intrusive.

Patients with DMD should be referred to respiratory/home ventilation teams for consideration of non-invasive ventilation (NIV) at the onset of symptoms of nocturnal hypoventilation and/or when the FVC is $<50 \%$ predicted.

\section{Lung volume recruitment and cough augmentation}

Other important aspects of care include lung volume recruitment (LVR) and cough augmentation. Respiratory muscle weakness leads to atelectasis and secretion retention as the inspiratory and expiratory muscles fail to generate effective inspiratory volumes and cough peak flows. LVR can be achieved via a ventilator, an LVR bag or Mechanical Insufflation/Exsufflation (MI-E). There are no clear values which predict ineffective cough in DMD, however a PCF $>2701 / \mathrm{min}$ when well, predicts an effective cough and low risk of developing respiratory failure during respiratory tract infections.

Historically, 'glossopharyngeal breathing' was used to facilitate lung recruitment. However, with the widespread availability of augmentation devices, this technique is now rarely used or taught.

An LVR bag can be used for recruitment or for secretion management. Its use in effectively mobilising chest secretions requires adequate expiratory muscle function.

Patients with DMD should be referred to respiratory/home ventilation teams for consideration of LVR 
or MI-E if they are experiencing chest infections, difficulty mobilising lower respiratory secretions or their PCF is $<2701 / \mathrm{min}$. An LVR bag is often used initially when $\mathrm{PCF}<2701 / \mathrm{min}$ or when there is difficulty mobilising secretions. Every complex home ventilation multi-disciplinary team should have access to MI-E provision when an LVR bag is not effective or when expiratory muscle weakness prevents effective cough.

\section{Other therapies}

Oro-pharyngeal secretion management may be required with a suction machine. Sialhorroea can be managed with antimuscarinic agents such as transdermal hyoscine patch, oral atropine, glycopyronium, propantheline or injection of botulinum toxin to the salivary glands. With recurrent chest infections assessment by a speech and language therapist is essential to exclude recurrent aspiration. The use of long-term antibiotics may be also considered when chest infections occur frequently. In patients with asthma or bronchial-hyperreactivity nebulised bronchodilators, steroids and ipratropium together with sputum management using mucolytics such as carbocysteine may all need to be considered.

Oxygen is not a treatment for respiratory failure and may further suppress respiratory drive although sometimes oxygen is required to be entrained into ventilator circuits e.g. to treat acute chest infection. Oxygen saturation targets in adult patients with DMD receiving supplemental oxygen should be $88-92 \%$ when not ventilated. Acute arterial oxygen desaturation is commonly caused by sputum plugging and retention in patients with marked respiratory muscle weakness. Intensive physiotherapy with use of cough augmentation techniques and NIV can help. If bronchial secretions are not excessive, then further clinical examination and investigation with chest $\mathrm{X}$ ray and blood tests are required to determine the cause of acute decompensation which may be due to acute chest infection, pneumothorax or pulmonary oedema.

\section{Respiratory multi-disciplinary team}

A holistic approach to therapy must be taken, with a good understanding of the individual's care arrangements and the impact on care provision that changes to their respiratory management (such as tracheostomy ventilation) might impose. The respiratory team should provide training for patients, families and carers including a competency assessment framework. A full range of ventilatory interfaces should be available including: nasal, full face, oronasal and mouthpiece. The multidisciplinary team (MDT) should anticipate and manage treatment complications such as ill-fitting masks, skin breakdown and abdominal distension. Tracheostomy ventilation may need to be considered where mask ventilation fails or when non-invasive ventilatory support is required for more than 16 hours continuously and is not tolerated by the patient or when respiratory secretion management with non-invasive strategies fails. Regular assessment of efficacy of respiratory support is essential with clinical review, oximetry, blood gases and overnight $\mathrm{TcCO}_{2}$ and $\mathrm{SpO}_{2}$ measurement where clinically indicated. Symptom management including palliative medicine and psychological support is important throughout the clinical course of the individual receiving ventilator support.

The respiratory support team is also responsible for equipment maintenance and should provide an emergency replacement service and 24-hour helpline. Patients requiring ventilation for $>16$ hours per day should have 2 devices both with internal battery support to ensure patient safety. The respiratory team should understand the arrangement for medical and social funded care packages for complex discharge planning from hospital to home environment. There should also be in place close liaison with the paediatric home ventilation service and there should be robust arrangements for "transition of care" into adult services. In addition, liaison with local hospitals, local respiratory physicians and intensive care units (ITU) is important when patients become acutely unwell. Other requirements of the service include wheelchair equipment mounting and other biomedical adaptations to equipment. Flight assessment before travel and the provision of backup equipment for holidays is also important. An understanding of advance care planning and the role of palliative care in supporting withdrawal of ventilation if requested/ indicated, is an important aspect for the respiratory team in conjunction with the neuromuscular team.

\section{Emergency admission to intensive care unit}

Sometimes a chest infection precipitates acute respiratory failure leading to invasive ventilation on intensive care. This can occur either before the institution of long-term respiratory support, or as an acute deterioration in a chronically ventilated patient. The prognosis for recovery is usually good, although the 
intensive care stay can be long, and patients usually have an increased need for long-term ventilation after the ITU admission. Patients with DMD who are admitted to ITU should have their management and weaning plan discussed with a respiratory physician responsible for the complex home ventilation service. Progression from mask ventilation to tracheostomy ventilation can have a profound effect on quality of life and care provision and may result in an ITU stay of many months.

Emergency admissions can be particularly stressful for patients with DMD and their families, especially if the admitting team are not familiar with the condition. An emergency care plan can be a useful document for the patient to carry and present on admission.

Key points

- Respiratory failure is inevitable in adults with DMD

- Assessment should be 6-12 monthly by experienced clinicians

- DMD adults are at risk of inspiratory muscle weakness, obstructive sleep apnoea, expiratory muscle weakness and aspiration

- Referral to a specialist home ventilation clinic is recommended even in asymptomatic patients whose FVC is $<50 \%$ predicted. Referral should be made regardless of FVC in the presence of symptoms of nocturnal hypoventilation

- Overnight respiratory monitoring to investigate nocturnal hypoventilation should be used to aid decision making especially regarding optimal timing of initiation of long term ventilation

- Refer to respiratory/home ventilation team for respiratory secretion management if: chest infections, difficulty mobilising secretions or $\mathrm{PCF}<2701 / \mathrm{min}$

- Every complex home ventilation service should have access to MI-E devices

- Anticholinergic medication such as hyoscine and glycopyrronium should be prescribed for sialhorrhoea/secretion management

- The respiratory MDT should support patients receiving ventilation offering a holistic and comprehensive service

\section{ANAESTHETIC CARE}

\section{Guidelines for perioperative care}

In general patients with DMD can be safely anaesthetised but there are significant potential complications which are related to pre-existing cardiorespiratory disease and the choice of anaesthetic agent [13]. Although guidance for professionals is important in this challenging area of anaesthesia, in general the safest anaesthetic is usually that with which the operative anaesthetist is most familiar and rigid guidance about technique is not appropriate.

Where practicable (outside of an emergency setting) patients with DMD undergoing General Anaesthesia (GA) or Procedural Sedation (PS) should be managed at a centre with significant experience in managing such patients and where there are intensive care facilities. Other than surgical experience, additional specialist experience required includes but is not limited to: anaesthesia, domiciliary ventilation services, cardiology and dietetics. Any patient having elective GA or PS should be referred to such centres. In the emergency situation, where transfer to specialist centres is not possible, advice should be sought from these centres.

\section{Pre-assessment}

All patients should attend an anaesthetic preassessment appointment as soon as surgery has been decided upon. Important issues to be addressed include:

1. 'Mouth opening', $26 \%$ of DMD patients have mouth opening $<40 \mathrm{~mm}$, significantly smaller than controls and can be challenging for an anaesthetist in terms of airway management [13-15].

2. All adults with DMD should be considered to have a cardiomyopathy. A consensus statement from the American Academy of Pediatrics, recommends cardiology review by a cardiologist with experience in managing DMD prior to any major surgery [16]. Cardiomyopathy is difficult to assess preoperatively due to the absence of symptoms and the difficulty in obtaining adequate echocardiographic windows in many DMD patients [17]. The information from a resting echocardiogram also provides limited information about cardiac function during significant physiological dis- 
turbances intraoperatively. Further testing with more dynamic tests (such as stress echocardiography) or cardiac magnetic resonance imaging (cMRI) should be considered [17].

3. Close liaison between the neuromuscular, cardiac, anaesthetic and respiratory services is essential and should take into account the patient's respiratory status prior to surgery, the magnitude (Minor, Moderate, Major) of the planned surgery and the patient's likely requirement for analgesia with respiratory depressant effects post-operatively. Patients can be divided into the following levels of dependency: Tracheostomy ventilated, Non-invasive ventilation (NIV)-dependent ( $>12$ hours per day NIV use), NIV overnight, not receiving NIV. The perioperative management in these situations is very different but in general, the first group is managed perioperatively with their usual tracheostomy ventilation. Patients on non-invasive ventilation prior to surgery require the input of the respiratory team. Usual practice would be to extubate the patient at the end of surgery directly onto NIV and continue this until the patient is fully awake and comfortable. NIV should then continue as normal post-operatively. The situation in patients who are not currently ventilated also requires close liaison between specialties. There are inconsistent data to suggest that in scoliosis surgery complications are higher in patients with an FVC $<30 \%$ predicted [18-22]. Usual practice would be to offer perioperative NIV to these patients with a set up some days or weeks prior to surgery to enable acclimatisation to NIV and direct extubation onto NIV following surgery to continue over the first post-operative nights. Previous guidance has suggested that patients with an FVC $<50 \%$ predicted should be considered for perioperative NIV [19], however, this would depend upon the magnitude of surgery and the requirement for analgesia with respiratory depressant effects post-operatively.

4. Nutritional status should be assessed prior to any procedure requiring GA.

5. When taking consent people with DMD should be made aware that GA or PS can be associated with a significant deterioration in respiratory status necessitating increased care requirements and therefore decreased autonomy. Specifically, patients who are NIV-dependent pre-operatively should be counselled about the possibility of respiratory failure and requirement for tracheostomy ventilation following major surgery. Patients should have access to adequate support preoperatively to help decide which surgical management option is right for them.

\section{Intraoperative care}

\section{Drugs}

1. Suxamethonium is absolutely contra-indicated due to the risk of hyperkalaemic cardiac arrest as well as triggering rhabdomyolysis [23].

2. Volatile anaesthetic agents have been associated with Anaesthesia-Induced Rhabdomyolysis (AIR) which is clinically and pathologically distinct from Malignant Hyperpyrexia (MH) [24]. There are case series of complications relating to rhabdomyolysis with the use of volatile anaesthetic agents including cardiac arrest. However, the majority of these reported patients were under the age of 8 years [24]. There are fewer reports of adverse outcomes at older ages which may be due to the reduction in muscle mass. The effect of steroids on these reports remains to be determined. It is rare in specialist centres not to have the expertise to manage DMD patients with total intravenous anaesthesia which would be the recommended practice. There may be certain situations where volatile anaesthesia may be useful such as the management of a difficult airway. A careful assessment of risks of, and alternatives to, volatile anaesthesia should be undertaken. If its use is considered unavoidable, once the airway is secured, the anaesthesia should be changed to an intravenous technique and the administration of volatile agent stopped.

3. Non-depolarising muscle relaxants are often not required during GA in DMD patients. Their use in DMD patients is associated with a prolonged duration of neuromuscular block [24-27]. However, the use of sugammadex to achieve complete reversal of profound neuromuscular block induced by rocuronium has been reported in a patient with DMD [28].

4. Many patients with DMD receive long term steroids and these should be given perioperatively and augmented during major surgery. 


\section{Airway management}

Airway management during GA has been successfully managed with endotracheal intubation, Laryngeal Mask Airway (LMA) and face mask. Endotracheal intubation remains the commonest technique due to the better control of minute ventilation. LMA use during gastrostomy formation is associated with a significant failure rate [29].

For PS, NIV has been used safely in patients on chronic NIV for diverse procedures, but its use has been most frequently investigated in patients undergoing gastrostomy formation [30]. It is a safe and effective technique but experience from specialist teams is that air leak during endoscopic gastrostomy formation is a significant problem and requires careful interface selection and monitoring as for a GA.

\section{Regional anaesthesia}

An expanding area of anaesthetic practice has been Regional Anaesthesia (RA). The successful use of RA (either neuroaxial or peripheral nerve blocks) has been reported either as a sole technique or combined with GA in ophthalmic, distal limb and orchidopexy procedures [31-33]. Consideration should be given to these techniques for intraoperative management and for postoperative analgesia.

\section{Cardiac output monitoring}

Invasive arterial pressure monitoring is recommended for all but the most minor of procedures. In procedures where significant blood loss is anticipated, cardiac output monitoring is frequently used to guide fluid requirements and replacement.

\section{Practical considerations}

Practical considerations for the anaesthetist include difficulty with intravenous access and with positioning of patients who may have significant joint contractures.

\section{Postoperative}

\section{Respiratory management}

NIV has a well-established role in the treatment of perioperative respiratory failure both in general settings [34] in patients with DMD undergoing spinal surgery [22] and in a cohort of patients with diverse conditions on chronic NIV undergoing multiple dif- ferent surgeries [34]. Early extubation is safe and practical following scoliosis surgery and should be the standard of care [18].

Although there are no data for the routine postoperative use of MI-E perioperatively, the treatment is well-tolerated [35] and has been shown to reduce hospitalisation rates and pulmonary morbidity in DMD patients [36]. Patients with a $\mathrm{PCF}<2701 / \mathrm{min}$ may benefit from postoperative use of MI-E therapy and preoperative training in its use.

All DMD patients undergoing GA or PS should be managed post-operatively in a critical care or high dependency setting for respiratory monitoring [35, 37].

\section{Fasting}

Prolonged fasting should be avoided in patients with DMD. Acute ketoacidosis has been reported in DMD patients with intercurrent illness even after only short periods of fasting [38]. Close monitoring and liaison with dietetic services is advised. If ketosis occurs an intravenous (IV) infusion of dextrose should be commenced [38].

Key points

- Patients requiring general anaesthesia should be managed in centres with expertise in the care of adults with DMD

- In the emergency setting where transfer is not possible, advice should be sought from a specialist centre

- There should be close liaison between the surgical, anaesthetic and respiratory teams

- There should always be a pre-anaesthetic assessment which should assess: airway (mouth opening), cardiac status (ECHO/ cMRI) and respiratory status

- At the time of consent patients must be informed of the risk of requiring long-term NIV or tracheostomy post procedure

- Suxamethonium is absolutely contraindicated

- A total intravenous anaesthetic technique is preferred due to the risk of anaesthetic induced rhabdomyolysis with volatile agents

- Non-depolarising muscle relaxants are not usually necessary and should be fully reversed before the end of anaesthesia

- Invasive arterial pressure monitoring is recommended 
- Early extubation onto NIV should be planned

- Prolonged fasting can result in ketosis, a dextrose infusion should be considered

\section{Cardiac management of adults with DMD}

This guidance builds on international standards of care [1-3] and the 238th international ENMC workshop for cardiac care [5] and has been adapted with an emphasis on the adult with DMD thus broadening the treatment options described in the SOC required to manage more advanced disease. Development of cardiomyopathy affects all DMD patients and most will have an established cardiomyopathy by 18 years of age [39]. Cardiomyopathy is one of the leading causes of morbidity and mortality [40]. Adults with DMD should be seen at least annually by a cardiologist with experience in the management of neuromuscular cardiomyopathy to monitor heart function, optimise drug therapies and provide feedback to patients. To achieve best long-term survival it is critical for paediatric and adult cardiology teams to liaise and agree thresholds for starting heart treatments, the drugs to be used and protocols for dosing. Cardiac care in adulthood cannot compensate for treatments not deployed appropriately prior to transition from pediatric services.

Assessment of cardiac symptoms in nonambulatory DMD adults is often difficult and limited. Even when left ventricular dysfunction is severely reduced, most patients remain asymptomatic and when heart failure ensues, symptoms are often non-specific with anorexia, abdominal pain and fatigue more often than shortness of breath predominating. Therefore, treatment and dose optimisation should not be based on the presence or absence of symptoms but needs to be guided by the serial results of cardiac imaging investigations.

Annual assessment by a cardiologist with imaging such as echocardiogram (echo) or cMRI should be undertaken, cMRI is the test of choice because it is better able to identify sub-clinical myocardial fibrosis at an early stage [5]. However, cMRI may be technically difficult in the adult patient and image quality reduced due to artefact from metallic spinal rods and inability of patients to lie flat for the duration of the scan. N-Terminal pro-Brain Natriuetic Peptide (BNP) measurement seems only to become elevated in patients with DMD with the onset of cardiac failure. As such, it is not clinically useful in monitoring the progression of ventricular dysfunction in these adults.

There is evidence to support the early initiation of an Angiotensin Converting Enzyme-inhibitor (ACEi) can reduce the rate of decline of cardiac function. Current clinical practice recommends empiric initiation of an ACEi prophylactically from 10 years of age and/or at a younger age when abnormalities are first detected on cardiac imaging [1, 5, 41-43]. A prospective randomised placebo-controlled trial in DMD patients showed that the mineralocorticoid receptor blocker, eplerenone, further reduces the rate of decline of cardiac function when added to ACEi therapy and its initiation is recommended in addition in patients with cardiac dysfunction [44]. Beta-blockers are also appropriate to slow sinus tachycardia and later when there is evidence of left ventricular systolic dysfunction because of their generic benefits in heart failure of diverse etiologies. However, evidence to support the use of a beta-blocker prophylactically in the early stages before the appearance of systolic dysfunction is lacking.

Therefore, the most evidence-based regime for managing cardiomyopathy in DMD adults is the combination of an ACE-inhibitor (ACEi), mineralocorticoid inhibitor (eplerenone / spironolactone) and beta blocker (BB) [41]. Most patients are likely to be already taking some or all of these medications before transitioning to adult services. However, doses will need to be optimised in line with patient size, weight and age changes over time thereafter. This three-drug combination is generally well tolerated. Up-titration of doses may be limited by low blood pressure in DMD patients, which appears to be prevalent [44]. Renal function should be monitored with U\&E and Cystatin C, especially, with the combination of an ACE-inhibitor and eplerenone [45, 46]. Patients need to be warned specifically about the need to always maintain an adequate fluid intake to avoid hypovolaemia, and especially so during periods of intercurrent illness [45, 46]. Renal failure due to acute kidney injury may occur in patients following addition of a diuretic to ACEi in the presence of dehydration [47]. Pragmatically, venesection is often difficult and may lead to delayed monitoring.

Unlike in most other forms of cardiomyopathy, glucocorticoid steroid therapy, used for skeletal muscle strengthening, delays the onset of cardiomyopathy by an average of two years. However, their role in treatment of cardiomyopathy in steroid-naive patients is not advocated [48]. 
Ivabradine may be used in selected DMD patients to slow sinus heart rate, particularly when a BB is contraindicated (such as in severe asthma) or as an adjunct to low dose BB when usual doses are not tolerated. The aim is to achieve a heart rate of between $50-70 \mathrm{bpm}$ as recommended in current heart failure guidelines. The evidence for use of ivabradine in this patient population is limited with only small trials showing some benefits [49].

The combination of sacubitril/valsartan ('Entresto') has been shown to confer significant symptomatic and survival benefits over conventional ACE-inhibitor therapies in patients with severe systolic dysfunction of other aetiologies although dosing may be limited by hypotension [50]. Currently, both Entresto and very recently licenced newer agents (SGLT2 inhibitors) [51] the utility and tolerability in patients with DMD are unknown, but may be considered in selected patients.

\section{Cardiac arrhythmias in DMD}

Atrial and ventricular arrhythmias may occur in DMD-related cardiomyopathy including atrial fibrillation or flutter with tachycardia which may aggravate pre-existing ventricular dysfunction. Treatment should be the same as for other causes of these arrhythmias in patients with other disorders who have similar degrees of LV dysfunction. Slowing ventricular response rates is important in preventing acute deterioration in LV function, culminating in overt heart failure symptoms [52, 53]. Although procedurally challenging, catheter ablation may be undertaken safely in adults with DMD with appropriate anaesthetic and respiratory support. Similarly, direct-current cardioversion may be indicated to restore sinus rhythm in those with atrial fibrillation precipitated by an intercurrent illness.

In adults with DMD, ventricular arrhythmias tend to track with the degree of LV systolic dysfunction. As such their impact on overall survival is unclear. Sudden unexpected deaths are described in patients with DMD but the extent to which they are attributable to arrhythmias is currently unclear and in need of further study. When sudden death occurs in an adult patient with advanced DMD it could be explained by profound bradycardia due to unheralded AV-block with ventricular stand-still, sustained ventricular tachycardia/fibrillation, pulseless electrical disease or pulmonary embolus $[5,54-56]$.

\section{Cardiac devices in DMD}

Implantable cardioverter-defibrillators (ICD) are indicated in patients with DMD who present with sustained ventricular tachycardia or who have survived cardiac arrest $[57,58]$. However, the benefit of prophylactic ICD insertion when there is severely reduced left ventricular function has not been established [59]. In addition, there is risk of complications at the time of device implant due to need for sedation or general anaesthesia and the extent of chest deformity, which increases the occurrence of pneumothorax, lead dislodgment, bleeding at access sites and infection [60]. Therefore, ICD use in adults with DMD should only be considered after thoughtful consideration and detailed discussion with the patient - involving carers/family. Ideally patients should be assessed and undergo operative intervention in high volume centres by an experienced operator.

Cardiac resynchronisation therapy with or without defibrillator capability (CRTP/D) should be considered in accordance with established guidelines, although the evidence for benefit in the context of DMD is only by extrapolation from randomised trials in cardiomyopathy of other aetiologies [61]. A registry of ICD or CRTP/D device implants in adults with DMD would help inform best practice in the long term.

Advanced heart failure treatment with mechanical support and heart transplantation is a theoretical option for patients presenting with severe heart failure. However, because of concomitant respiratory and skeletal muscle weakness, in practice, patients with DMD will rarely be assessed as eligible for these therapies. However, a small number with milder intermediate (BDMD) phenotypes have been transplanted in the USA.

Anticoagulation for systemic thrombo-embolic prophylaxis may be considered in patients with atrial tachyarrhythmias and in those with severe left ventricular systolic dysfunction to reduce their risk systemic thrombo-embolus. Those considered at particular risk of venous thrombosis with pulmonary embolism may also warrant anticoagulation. The use of novel oral anticoagulants (NOAC) is preferable to vitamin $\mathrm{K}$ antagonists for patient convenience, ease of dosing and to reduce the need for repeated INR (international normalized ratio) checking needed with warfarin.

Finally, timely initiation and optimisation of NIV is also supportive for the heart in DMD patients and 
is a key part of the overall strategy for improving cardiac outcomes cardiac outcomes [55].

\section{Specific drugs \& doses}

There is lack of evidence on most appropriate doses of cardio-active medications to use in the early early subclinical stages of DMD-cardiomyopathy. At the stage, cardiac fibrosis may be seen on cMRI, global systolic function is usually still within the normal range. Studies on prophylactic use of ACE- inhibitors in this patients' population have left dosing to the discretion of the clinicians. However, by extrapolation from the results from heart failure studies, it seems logical to up-titrate the doses to the maximum tolerated to achieve better results. For patients with reduced systolic function, generic guidelines for use of drugs blocking the renin-angiotensin-aldosterone system and beta-blockade in heart failure of commoner etiologies are appropriate. Drugs should be initiated and up-titrated as tolerated [62-67]. There is need for more experience in using sacubritil/ Valsartan, in place of either an ACEi or Angiotensinreceptor blocker and/or SGLT2 inhibitors in patients with DMD cardiomyopathy to make recommendations.

Key points:

- All patients should have annual cardiologist follow up with assessment of cardiac function by echo or cMRI.

- cMRI should be considered at baseline to detect early pre-clinical cardiac involvement and guide treatment

- Combinations of ACEi, beta blockers and mineralocorticoid inhibitors slow the rate of decline in cardiac function in DMD

- All patients should have been started on treatment before transition, but if not, treatment with ACEi, beta-blocker should be started, a mineralocorticoid inhibitor should be initiated in patients with systolic dysfunction. Doses should be up-titrated to the maximum tolerated

- Careful attention to fluid intake and regular monitoring of renal function is important, especially, during intercurrent illness and if adding a loop diuretic.

\section{Renal and bladder management}

Renal function can be compromised in adults with DMD, usually as a result of progressive cardiac failure and its treatment [56, 57]. Renal dysfunction is associated with a poor prognosis with $14 \%$ allmortality from acute renal failure in DMD and was first reported in Japanese patients [68]. There is no specific guidance for managing renal complications in DMD in the published literature including international SOC [1-3]. The National Institute of Clinical Excellence (NICE) adult chronic heart failure guidelines recommend regular monitoring of renal function [56] as renal failure is an independent risk factor for mortality and adverse cardiovascular event in adults with heart failure [69].

\section{Risk factors}

Cardiac dysfunction and its treatment with ACEi, beta blockers and diuretics impact on end organ function. Patients with DMD have additional risk factors including reduced fluid intake, and steroid side effects [1]. Braat et al. prospectively identified a progressive decline in GFR in DMD children and adolescents with increasing age [70]. Chronic untreated hypertension is another risk factor in corticosteroid treated patients, in one series, $50 \%$ patients had a non-dipping BP profile (nocturnal decrease $<10 \%$ of daytime BP) [60]. Hypertension and non-dipping blood pressure support the hypothesis that the reninangiotensin aldosterone system is active in DMD [71]. Reports of renal failure in DMD demonstrate common features including heart failure, chronic decreased fluid intake and prolonged use of diuretics [72-75]. Non-ambulatory DMD patients are at risk of reduced kidney perfusion, which leads to pre-renal failure. In adults with DMD, normally the serum creatinine is very low as a consequence of very low muscle bulk. Thus, in DMD patients when there is renal impairment, the serum urea is markedly raised and the creatinine increases significantly above the baseline value for that patient, although it is still most likely to be within the normal range. This is due to the very low serum creatinine levels normally seen in DMD. As a consequence, Cystatin C is a useful additional test for detecting early renal impairment in DMD [73-75]. Anaemia (normocytic, normochromic) often accompanies kidney failure and should always trigger investigation of renal function [76].

Monitoring full blood count and renal function using urea and electrolytes (U\&E) and Cystatin C at least annually is recommended for all DMD adults. 
If an abnormal result is found, then a Glomerular Filtration Rate (GFR) scan and urgent referral to a consultant nephrologist is recommended.

Anaemia is associated with renal failure and the effect can be cumulative due to increased cardiac workload and renal hypoperfusion, some patients may require treatment with erythropoietin. Encouraging adequate fluid intake in all DMD patients is important to maintain renal perfusion [68].

Acute gout occurred in one adult patient belonging to the network who was known to have severe end stage cardiac failure (ejection fraction 10\%) and renal impairment (Cystatin C $2.2 \mathrm{mg} / \mathrm{l}$, normal range $<1.0 \mathrm{mg} / \mathrm{l})$. He presented acutely to Accident and Emergency with a painful swollen knee and subsequently elbow. He had raised inflammatory markers and serum urate was 1000umol/l (normal range 200-430 umol/1). Management included corticosteroids during the acute phase followed by allopurinol once the symptoms had settled. Hyperuricaemia and gout were thought to have been caused by a combination of diuretic treatment and renal impairment secondary to cardiac failure.

\section{Renal calculi}

Renal calculi can be a troublesome problem affecting about $10 \%$ adults with DMD [77]. They probably occur secondary to increased urinary calcium excretion, although measurement of urine calcium creatinine ratio is impossible due to the low muscle mass and low serum creatine level. Patients affected with renal calculi may experience pain and hydronephrosis necessitating analgesia and drainage with nephrostomy, which in turn poses additional risks for the adult with DMD. Voluntarily limiting oral fluid intake is not uncommon in older DMD patients. It is important to assess fluid intake at every clinic visit and to encourage the patient to drink 1-2 litres of fluid each day. Currently, there is insufficient evidence to support annual renal ultrasound to screen for calculi.

\section{Bladder dysfunction}

Bladder dysfunction is also reported amongst people with DMD and can be a distressing symptom. A questionnaire survey showed that some patients had symptoms of daytime incontinence, urinary frequency, urgency, nocturnal enuresis, nocturia, stress incontinence and urinary hesitancy [78]. Urodynamic studies showed a small capacity, hyperreflexic blad- der and detrusor sphincter dyssynergia [79]. Detailed history and examination are important to exclude other causes of urinary dysfunction. Postural changes may help with voiding, some patients reported that using a neoprene strap to reduce their lumbar lordosis helped them to micturate. If symptoms are suggestive of detrusor hyperreflexia, a pre and post micturition bladder ultrasound to ensure complete bladder emptying and a trial of oxybutynin or a similar medication can be considered. The use of chronic indwelling catheterisation has been practised for some patients, although infection and leakage may be troublesome.

Key points

- Baseline serum creatinine is usually very low in DMD

- Annual monitoring of renal function is recommended (urea, electrolytes and Cystatin C). In renal failure, urea and Cystatin $\mathrm{C}$ will be abnormally raised. Creatinine will be raised above baseline but is likely to be within the normal range

- Anaemia may be a sign of renal failure

- Consider gout due to hyperuricaemia in the context of acute joint swelling in patients on diuretic treatment for heart failure

- Renal calculi are common in DMD, measuring urinary calcium:creatinine ratio is not helpful due to the low serum creatinine levels

- Ensure all adult DMD patients have adequate fluid intake

- Consider treatment with medication such as oxybutynin or tolderodine for symptoms of sphincter dyssinergia

- Postural manoeuvres to reduce the lordosis may help with voiding

\section{NUTRITION AND GI COMPLICATIONS}

As outlined in the international SOC [1-3], weight and nutrition should be assessed every 6-12 months in adults with DMD. Those patients on long term corticosteroid treatment are at risk of obesity leading to increased problems with metabolic syndrome and obstructive sleep apnoea. In addition, obesity makes transferring and hoisting more difficult. Regular dietetic advice on weight management is important 
[1]. Where there is metabolic syndrome, treatment with metformin may be considered [80]. On the other hand, while obesity is a problem in children and younger adults, most adult patients are likely to develop dysphagia and difficulty in chewing, this in combination with not being able to self-feed can result in marked weight loss and cachexia [81]. Cachexia may increase the risk of infection and pressure areas and may be associated with a worse outcome. Thus, regular weight monitoring, assessment of swallowing and oral intake is essential. Referral for assessment by a speech and language therapist and dietitian should be made if the patient is losing weight or reporting dysphagia. Ideally such therapists should be embedded within the clinical team, to facilitate early identification and intervention for these complications.

The patient support group, DMD Pathfinders has developed an advice guide to managing nutrition which includes daily living tips and recipes to help people with DMD in various stages of dysphagia: (https://dmdpathfinders.org.uk/resources/adviceguides/).

Recurrent weight loss requires input from a dietitian and if weight cannot be maintained with or without dietary supplements a percutaneous gastrostomy (PEG) should be considered and discussed with the patient and his family [82, 83]. Where there has been significant weight loss, a careful program to prevent 're-feeding syndrome' is essential [84]. Re-feeding syndrome, which is not described in the standards of care [1-3], is a potentially lethal complication caused by shifts in fluids and electrolytes in a cachectic patient being treated with enteral or parenteral nutrition. This regimen of care should be carefully co-ordinated by a gastroenterologist and expert dietitian.

Adults with DMD have significant GI dysmotility and, as a consequence, frequently experience constipation and abdominal bloating that may be so severe that it can be life-threatening and impact on breathing and ventilation. Constipation may also result in painful anal fissures that have a significant impact upon quality of life. Constipation may also lead to diverticulitis and diverticular abscesses. Thus, treating and avoiding constipation is very important in the management of adults with DMD, it is a symptom that is frequently overlooked [96, 97].

Chronic mild abdominal bloating can sometimes improve with dietary measures by avoiding certain foods such as gluten, onions, beans, pulses etc.
[80]. Recurrent severe bloating with abdominal pain should be investigated with abdominal $X$ ray to exclude volvulus which has been reported in adults with DMD) [98-100]. Episodic abdominal bloating may result from small intestinal bacterial overgrowth (SIBO), which can be identified with a breath test and treated with antibiotics. Identifying SIBO may need the input of a gastroenterologist or expert clinician [91]. Gaseous distension may be helped by venting via the gastrostomy tube or with a rectal 'flatus' tube, the latter of which needs to be inserted with caution [92]. Regular osmotic laxatives, such as macrogol, should be considered early on, with or without the addition of a stimulant such as senna or bisacodyl. Enemas should be used with caution in the frail cachectic patient, but suppositories may be used to improve voiding without needing to strain. Currently there is no evidence to support colonic irrigation or stoma formation to treat constipation in DMD, both of which potentially carry a high risk to the adult patient.

Pilonidal sinus has also been reported amongst the network patients and may lead to osteomyelitis. Increased hair growth, poor hygiene and sweating due to rubber wheelchair cushions have been suggested as possible causes.

Gastro-oesophageal reflux (GOR) may cause unpleasant symptoms in some patients, especially those on corticosteroid treatment who are obese and also in those with scoliosis, the use of positive pressure ventilation may also increase the risk of GOR [91]. In addition, patients with DMD have slowed gastric emptying, which may worsen over time and symptoms may be similar to GOR including abdominal pain, nausea, vomiting and a feeling of fullness. Management may include: low fat diet, avoiding meals immediately before recumbency, frequent small meals, sleeping more upright and prokinetic drugs (paying attention to the potential QT prolongation effect of some of these) and low volume PEG feeds and flushes. When severe, jejunal feeding may be required. Antacids with aginate (such as peptic or gaviscon) can be helpful to relieve symptoms- they can sometimes alter bowel function, which should be treated as appropriate. Blocking acid with $\mathrm{H} 2$ receptor antagonists and proton pump inhibitors is an option, but the possible long term adverse effects have not been fully explored such as the increased risk of fractures due to osteoporosis [81] and the former are difficult to prescribe now. For those patients taking corticosteroids, a gastroprotective preparation should be used. 
Key points

- GI symptoms are common in adults with DMD, actively treat and prevent constipation

- Weight management includes preventing both obesity and cachexia, involve a dietitian and speech and language specialist

- Consider Metformin for obese patients with metabolic syndrome

- Re-feeding syndrome following significant weight loss can be fatal, there should be a plan in place to prevent this when feeding support is initiated.

- Abdominal bloating can be life threatening, consider venting via gastric and rectal tubes, inserted with caution

- Certain dietary measures can decrease abdominal bloating

- Consider prokinetic agents for gastrooesophageal reflux

\section{Corticosteroid management, endocrine and bone health}

The adult DMD population is heterogeneous including patients who are: a) steroid naive (defined as either no treatment or treatment $<1$ year), b) previously steroid treated but discontinued ( $>1$ year treatment) and c) those still taking steroids either daily or intermittently (10 days on 10 days off). If the DMD adult has benefitted from steroid treatment- as evidenced by comparison with age-matched steroid naive peers, and benefit continues to outweigh potential harms, the patient should be given the choice to continue treatment. Such benefits might include: better muscle function (as evidenced by: ability to walk, stand, crawl or sit without support), positive effect on upper limb function and preservation of respiratory and cardiac function.

Adult patients who are taking steroids are at risk of steroid related complications that require regular monitoring which, as outlined in the standards of care [1-3] include: hypertension, cataracts, glucose intolerance, obesity, infections, short stature, friable skin, GI and bowel perforation and pubertal delay due to hypogonadism, osteoporosis, adrenal insufficiency and fat embolism as a consequence of osteoporotic fractures. If these complications outweigh any perceived benefit in the adult patient, then the patient should be given the option to wean and stop steroid treatment. Weaning should be done very slowly and the patient warned that he is at risk for adrenal insufficiency for at least 12 months post weaning.

Adult DMD patients on steroids who develop Herpes Zoster infections should be treated promptly with oral or IV acyclovir.

\section{Bone health}

These consensus guidelines on bone health are adapted from the ENMC 236th workshop on bone protection in DMD [4] and the international standards of care [1-3]. Actively manging bone health is important for all DMD patients. Fractures are common in those who remain on corticosteroid therapy, although muscle weakness itself is also a risk factor for poor bone health. Long bone fracture prevention is important because of the risk of fat embolism syndrome (FES), a rare life-threatening complication so far described in paediatric DMD patients. Sudden onset of breathlessness, cyanosis, rash, and confusion occurring soon after long bone fracture should raise suspicion of FES which requires urgent critical care [93].

All patients should be regularly monitored in conjunction with a metabolic bone specialist. The international SOC recommend that patients on long term steroid treatment should be assessed annually for symptoms and signs of steroid induced osteoporosis with a lateral spine imaging and DXA scan [1], although lateral spine imaging should be prioritized. There are however challenges in performing bone monitoring investigations in the adult population.

Lateral thoracolumbar spine imaging aims to identify vertebral fractures which are very common in DMD, especially in those treated with steroids. The presence of vertebral fracture signifies significant bone fragility and when present, treatment with bone protective therapies (such as a bisphosphonate) is indicated, regardless of the bone mineral density. Lateral spine imaging can be conducted using standard $\mathrm{X}$ rays of the thoracic and lumbar spines or with Vertebral Fracture Assessment (VFA) using DXA. Back pain, in particular lower back pain, is very common in adults with DMD [94] which may be due to reasons other than fracture. However, any new and severe back pain including in those who are on corticosteroid should prompt imaging of the spine to look for presence of vertebral fractures. In patients with new and severe back pain, consideration of other modalities of imaging the spine (for example CT or MRI) maybe needed to diagnose or rule out vertebral frac- 
tures if $\mathrm{X}$ rays are normal, as $\mathrm{X}$ ray imaging may not be of sufficient quality in adults with DMD due to significantly osteopenic appearance of bones on $\mathrm{X}$ rays and/or due to scoliosis. Lateral spine imaging and DXA is problematic in adults with metal instrumentation for scoliosis and should be individualized. DXA bone density scans of the hips may give some information but may often not be feasible in these adults as contractures make positioning required for the scans extremely challenging. The majority of men with DMD on corticosteroids treatment have varying degrees of short stature. This impacts on interpretation of DXA bone density and requires adjustment for size. Height measurement is a challenge in these adults, and size adjustment of DXA bone density is not routinely performed in adult DXA services. DXA monitoring is needed if the patient is on bone protective therapies like Bisphosphonate but it is anticipated that an individualized plan should be made in conjunction with a metabolic bone specialist.

A vitamin D supplement should be given to all patients. Serum $25 \mathrm{OH}$ vitamin D should be checked annually and levels maintained above $50 \mathrm{nmol} / \mathrm{L}$, anecdotally, most paediatric DMD clinics try to maintain the level above $75 \mathrm{nmol} / \mathrm{l}$, although there is no evidence from trials. Attention should be paid to dietary calcium to ensure that it is adequate.

All patients with a history of low trauma long bone fractures in particular femur and humerus; and those with evidence of vertebral fractures identified on lateral spine imaging even if asymptomatic should be considered for treatment with bone protective therapies. Options include anti-resorptive therapies with bisphosphonate (intravenous or oral) and RANK ligand antibody, Denosumab (subcutaneous injections); or anabolic bone protective therapies like Teriparatide and Romosozumab [4]. There are no published data on the efficacy of any of these bone protective therapies in adults with DMD; and each may come with some risks, hence the need for individual discussions with a metabolic bone specialist. Such therapies should be initiated by a metabolic bone specialist with input for ongoing monitoring. For all patients receiving bisphosphonate therapy and RANKL ligand antibody therapy, dental hygiene and regular monitoring is important because of a potential risk of osteonecrosis of the jaw.

\section{Adrenal insufficiency}

Treatment dose higher than Prednisolone $5 \mathrm{mg}$ (or Deflazacort $6 \mathrm{mg}$ ) daily for as short as 4-6 weeks in adults is known to cause secondary adrenal suppres- sion in adults.

Treatment dose of corticosteroid in paediatric patients with DMD is at least three times higher than the dose that would cause secondary adrenal suppression in an adult if used for as short as 4-6 weeks. Therefore, all adult DMD on corticosteroid therapy have secondary adrenal suppression, which is potentially life-threatening, as they would have been treated $>10$ years by the time they transition to the adult clinic. All patients on corticosteroids should be aware of the risk of an adrenal crisis, which is potentially life-threatening, and when to seek medical attention $[1,6]$. They should be advised to never allow their supply of medication to run out nor should they suddenly stop treatment. An alert should be in place in the hospital for appropriate steroid cover during emergencies and surgical procedures. Adult men with DMD should be encouraged to carry steroid identity cards or bracelets.

During acute illness or planned surgery, patients who have been on long-term steroid treatment are at risk of an adrenal crisis. In the UK guidance is available via the Society for Endocrinology [6] in which it is recommended that patients on physiological replacement of corticosteroids (e.g. Addison's Disease) should double their usual steroid dose during periods of illness (such as those requiring antibiotics). However, adults with DMD are on supraphysiological doses of corticosteroid. Almost all men with DMD on corticosteroid will be on doses which are sufficient for mild acute illness. However, it may be advisable to split corticosteroid dosing during acute illness to ensure sufficient cover on a continuous basis. This may be particularly an issue for those on Deflazacort as the plasma elimination half-life is 1.1 to 1.9 hours, with little published information on its biological half-life. The plasma elimination half- life of Prednisolone is approximately 2 to 4 hours, with biological half-life of 18-26 hours. As an example, a man who is normally on Prednisolone $20 \mathrm{mg}$ daily can be advised to take prednisolone $20 \mathrm{mg}$ in the morning and $20 \mathrm{mg}$ in the evening during acute illness. Another option for management of steroids during mild to moderate acute illness is to instruct patients to take oral hydrocortisone $20 \mathrm{mg}$ 3-4 times a day in addition to usual Prednisolone or Deflazacort dose. It is anticipated that local and national policies should be followed in discussion with the local endocrinology team.

In case of vomiting illness, patients should be advised to repeat the oral dose of steroid after one hour. If vomiting continues or the person becomes 
unwell (particularly if there are symptoms to suggest adrenal insufficiency), the patient should be advised to go to hospital. It is recommended that patients should have access to injectable hydrocortisone at home for administration prior to going to hospital. Families or carers should be trained to give IM Hydrocortisone $100 \mathrm{mg}$; alternatively, it can be administered by paramedics.

There is often an acute phase response after initial treatment with IV bisphosphonates for steroid induced osteoporosis. It may be wise to increase the usual steroid dose to cover the first infusion.

\section{Pubertal delay and hypogonadism}

One adverse consequence of steroid treatment is pubertal delay and hypogonadism. The majority of adolescents on corticosteroids especially daily regime will not commence puberty spontaneously and will require testosterone therapy to aid completion of pubertal development, improve linear growth and restore bone accrual closer to those of a healthy adolescent. Testosterone therapy is discontinued once pubertal development is complete. Some young men with DMD continue to have central hypogonadism (low testosterone levels, with normal or low LH and FSH). Untreated this may impact on bone health, fatigue and energy levels. Monitoring testosterone levels together with LH and FSH is recommended at least once every 2 years. Ideally, testosterone levels should be checked in the morning close to 9 am, if possible. Discussion with endocrinologist for treatment with testosterone therapy is indicated if low testosterone levels is identified on two occasions [1], and this should be managed with input from an endocrinologist.

\section{Glucose intolerance and metabolic syndrome}

There is limited but growing research evidence for abnormal glucose metabolism in DMD adults, this could be related to corticosteroid treatment and obesity, but might also be related to loss of muscle tissue that would otherwise be involved in glucose metabolism [94]. Clinical assessment should look for evidence of metabolic syndrome and the finding of acanthosis nigrans should prompt testing of $\mathrm{HbA} 1 \mathrm{C}$ and if abnormal appropriate treatment commenced under the guidance of an endocrinologist. Where there is evidence of metabolic syndrome metformin may be prescribed. Acute episodes of normoglycaemic ketoacidosis have been reported recently in adult DMD and should be managed with IV fluids [95]. Further prospective natural history data, as planned by the ANSN will add to our understanding of these rare metabolic complications in the future.

Key points

- Corticosteroids should be continued if evidence for benefit outweighs risks

- Monitor corticosteroid side effects regularly

- 25OH Vitamin D should be maintained $>50 \mathrm{nmol} / 1$

- Osteoporosis should be managed by a metabolic bone specialist

- Fagility fractures should be treated with a Bisphosphonate and/or RANK ligand

- All steroid treated patients should have a plan in place to manage adrenal insufficiency during acute intercurrent illness

- Testosterone levels should be monitored and treated when deficient

\section{Transition}

The transition process should begin in the pediatric service during adolescence to prepare the young person for adulthood, this is discussed in the international standards of care [1] and there are NICE guidelines which should be followed (https://www.nice.org.uk/guidance/ng43). There should be joint clinics between the pediatric and adult physicians to facilitate a smooth transition and transfer to adult services. There should be a transition coordinator to set goals with the young person and to support them during adolescence and teenage years. The young person should have a voice and be heard. Providing peer support through the service and/or directing the young person to a peer support group via a national charity is also advised.

\section{Participation, psychosocial, and palliative care}

Comprehensive multidisciplinary care of adults with DMD should include access to therapy services including: physiotherapy, speech and language therapy, occupational therapy, clinical psychology and a care advisor. A care advisor or equivalent (such as Clinical Nurse Specialist) can play a major role in supporting participation at all stages of the condition. At times, orthopaedic surgery and chronic pain 
teams may also need to be involved. Musculoskeletal pain can a frequent symptom in adults with DMD, strong opioid analgesics may exacerbate constipation and bowel issues. Gabapentin is a useful adjunct and may be considered but early referral to a pain team for localised injections can be very helpful.

The role of the care advisor is outlined in Table 1

\section{Participation in activities of daily living for adults with Duchene Muscular Dystrophy (DMD)}

A primary goal of the care of the adult with DMD should be to facilitate participation. Participation is defined as direct engagement in a life situation including activities of personal care, mobility, social relationship, education, recreation and leisure, spirituality and community life. It is considered a measurable outcome of health and is increasingly becoming a key focus of rehabilitation programs [96].

For the general neuromuscular population decisions about occupation and employment are often dictated by physical function, muscle strength, fatigue and type of neuromuscular condition [97]. Not surprisingly, unemployment and social isolation are frequent complaints [98, 99]. Qualitative studies examining barriers to meaningful occupation in adult DMD patients indicate lack of support and resources, social isolation, lack of motivation and depression in addition to medical challenges [100]. Fatigue, pain and affective disorders may be more prevalent in the older DMD population [101] and these likely further impact upon participation in adulthood.

It is likely there is an interaction between physical health, psychological factors and societal factors. For young men with DMD and their families the disease impacts on all three areas with dramatic changes in lifestyle and functional performance and increasing loss of physical function and independence. This follows a pattern observed in younger DMD patients [102].

Outcome measures for persons diagnosed with DMD have, over the years, been mainly focused on the assessment and monitoring of disease progression. The emphasis has naturally been to focus upon features such as muscle strength and contractures, respiratory and cardiac involvement and other important medical aspects. However, these clinical measures may not correlate directly with daily life activities and quality of life issues which an individual experiences and aspires towards. There is a need to establish a more complete assessment of participation in daily life activities and for disease management to be examined in the context of valued participation in real life situations.

To date, this has been studied mainly in the paediatric DMD population using for example the Children's Assessment of Participation and Enjoyment (CAPE) and Paediatrics Quality of Life Inventory (PedsQL) scales [103] and the ACTIVLIM questionnaire [104]. Studies in the adult Duchenne population have used narrative interviews [100, 103], SF-36 and WHOQOL-BREF [105-107]. Scales used in the adult population focus predominantly on quality of life evaluation rather than actual detailed performance participation. There is a need to assess these aspects and impact on quality of life in order to address the needs of adult men with DMD including to: identify and evaluate scales used in adult patients with neuromuscular diseases and gain consensus on which scales to use. Currently available scales include: MAPA - The Meaningful Activity Participation Assessment which is a checklist-type survey of 28 diverse activity items assessing frequency of participation and degree of personal meaningfulness experienced with each activity, assessment of pain, ACTIVLIM, QoL: SF36, WHOQOL-BREF. Assessing factors that impact on participation and quality of life also need to be considered. These include assessing pain, fatigue and mood. The Fatigue Severity Scale is a 9 item scale with good psychometric properties [108]. The Hospital Anxiety and Depression Scale [109] is a well-known screening measure. Higher scores indicate 'caseness' for depression or anxiety and warrant further assessment by mental health professional or referral to psychological therapies. These measures can be used routinely by care advisors or clinical nurse specialists to flag up individuals who may need further intervention.

\section{Psychological care}

Evidence for cognitive impairment in young DMD patients is well established [110-113]. Deficits have been reported in verbal and visual memory, working memory, executive function and higher cognitive processes attentional processes. Neuropsychiatric disorders including ADHD, autism or autistic spectrum disorder and obsessive compulsive disorder (OCD) are also prevalent [114]. Whilst some cognitive difficulties may resolve with age [115] there is evidence that difficulties in general information processing persist into adulthood [116] as do autistic traits and/or Attention Deficit Hyperactivity Disorder (ADHD) and OCD [117]. 
Table 1

The role of the care advisor in supporting participation

\begin{tabular}{|c|c|}
\hline Activities & Specific actions \\
\hline MEDICAL & $\begin{array}{l}\text { Facilitating referrals } \\
\text { - MDT involved in health care } \\
\text { Care planning } \\
\text { - Assessment of need to support patients to make decisions/choices } \\
\text { - Formulating individualised care plans - transition, advance, emergency, palliative } \\
\text { Genetic counselling } \\
\text { - Ensuring that there is appropriate referral, signposting for families to genetic screening } \\
\text { - Support to understand the complications and potential outcomes regarding genetic counselling } \\
\text { - Follow-up support following genetic counselling } \\
\text { Clinics } \\
\text { - NM advisor to be present at clinics } \\
\text { Hospital admissions } \\
\text { - Improve patient experience during admission } \\
\text { Discharge planning } \\
\text { - Liaising with CHC, appropriate level of care to reflect change in needs following admission } \\
\text { Education } \\
\text { - Clinical staff in contact with NM teams in a timely manner } \\
\text { - Education of clinical and therapy staff about NM care } \\
\text { - Co-ordination of care } \\
\text { - Appropriate condition specific info in place - alert cards, emergency care plans, palliative, etc }\end{array}$ \\
\hline SOCIAL CARE & $\begin{array}{l}\text { Housing } \\
\text { - Identify future need and make appropriate referrals to reduce delays and reduce costs to local } \\
\text { authorities. } \\
\text { - Providing links to expert resources regarding adaptations } \\
\text { - Advice regarding re-housing needs - specific needs around future proofing in accessible home } \\
\text { environment } \\
\text { Respite care } \\
\text { Personal health budgets } \\
\text { - Liaise with providers and commissioners with condition specific info to support provision of } \\
\quad \text { effective care, } \\
\text { Disability benefits } \\
\text { - Support access to condition specific information to support applications through to tribunal } \\
\text { Higher education } \\
\text { Disabled students grants } \\
\text { Employment } \\
\text { - Advice re work } \\
\text { - Occupational health } \\
\text { - Access to work - environmental adaptations } \\
\text { Leisure } \\
\text { - Signposting, assisting with grant applications for specialist equipment and supporting social care } \\
\quad \text { pathway. }\end{array}$ \\
\hline SELF-MANAGEMENT & $\begin{array}{l}\text { Education } \\
\text { - Educate patient, families and carers about condition and early signs, symptoms, parameters } \\
\text { trigger points for seeing medical advice and access to support }\end{array}$ \\
\hline THERAPIES & $\begin{array}{l}\text { Equipment \& technology } \\
\text { - Referrals to environmental control } \\
\text { - Assistance with applications for specialist equipment not provided by local authority and NHS } \\
\text { trusts } \\
\text { - Information }\end{array}$ \\
\hline PSYCHOSOCIAL & $\begin{array}{l}\text { Emotional support } \\
\text { - Holistic approach to support family } \\
\text { - Transition to adult services } \\
\text { - At changes of function (respiratory, cardiac, mobility) } \\
\text { - Close links with palliative care services (acute \& community). Knowledge of palliative care needs } \\
\text { - End of life - time limited support for carers post bereavement }\end{array}$ \\
\hline
\end{tabular}


Table 1

(Continued)

\begin{tabular}{ll}
\hline Activities & Specific actions \\
\hline TRANSITION & Co-ordination \\
& - Co-ordinating healthcare from paediatric services to adult services and not lost \\
& - Support for young people as well as their families and carers \\
- & Safeguarding \\
- GP & \\
Education & Education, support and co-ordination for young people as well as their families and carers - using \\
& a framework such as Ready Steady Go. \\
& Increase independence in managing condition through education \\
\hline
\end{tabular}

Psychological disorders such as depression and anxiety are also associated with DMD with rates ranging from $17-29 \%$ for depression and/or anxiety $[118,119]$. Depression and anxiety may become more prevalent in adulthood as the young person grapples with adjustment to a progressing health condition $[118,119]$.

Consideration also needs to be given to the impact of these changes on the support system and family. Parents face the burden of providing greater levels of care as well as the social and psychological support for their children [120]. Assessing carer burden and the presence of anxiety or depression in parents may also be helpful.

The adult DMD patient and their family face a number of challenges from the medical, social and psychological domains. The interaction of these different factors and how they are managed can have a bearing on treatment compliance; especially NIV use and/or cardiac medication which can have lifethreatening complications.

A clinical neuropsychologist (or equivalent) should be part of the neuromuscular multidisciplinary team (MDT) and play a role in screening and risk assessing individuals where appropriate. Anti-depressant and anxiolytic medication should be considered for those people with moderate to severe symptoms. Referral for psychological intervention may also be useful. Intervention can range from individual or group treatment for specific concerns such as anxiety and/or depression, adjustment. Neuropsychological review should also be considered to provide a baseline of cognitive function and highlight weaknesses that may impact on the patient's ability to manage educational and work activities as well as self-care and decisions around their health condition and treatments.

\section{Palliative care}

This is a new group of patients for palliative care and the trajectory of their condition is not as clear cut as patients with other life-limiting diseases such as cancer for example. However, it does fit with the change in the palliative care ethos with an emphasis on patients with non-malignant conditions rather than cancer and an emphasis on symptom control as well as end of life care [121]. Any chronic condition, particularly when life-limiting, can lead to psychological adjustments and the fear of dying and how that process will happen [122].

There is a very limited body of literature on palliative care and 'End of life' discussions in adults with DMD and the majority highlight that good quality end of life discussions are not usually held by the clinical team; DMD men may have questions about what the end of life will be like [122]. In one study $85 \%$ of families had not heard of the palliative care team and hospice care only in $6 \%$. Only $25 \%$ had any form of advance care directive document [123].

Involving a palliative medicine consultant in patient care is helpful. Palliative medicine consultants are often embedded within the hospital setting, where this is not the case a 'hub and spoke model' with the neuromuscular team should be considered. The palliative medicine consultant can be called upon to help with symptom control and in the utilisation of a local palliative care service. All patients should be able access a palliative care consultant at any time during the course of their condition. Advance care plans in general medical care are now widely used and accepted. The application within DMD is difficult- both for when and how to introduce this topic of discussion and by whom should this discussion be initiated. Current experience suggests that it 
is the team who knows the patient best who should do this. There is also a perception that advance care plans are only about end-of-life discussions when they should be recognised as a means of documenting the patient's wishes in terms of 'ceiling of care' and priorities. A recent review by Hiscock et al. revealed that majority of DMD patients die without a formal end of life care plan in place [124]. There appeared to be a shared reluctance of patients, family carers and healthcare professionals to initiate these discussions. Discussing end of life issues is an uncomfortable area for doctor patient discussions, an ENMC workshop on adult DMD care heard from participating people with DMD that they did not wish to have end of life discussions [125]. On the other hand, in a study by Abbott, young DMD men expressed a desire for clinicians to be proactive in their approach to bring up these topics and discussions [123]. Such discussions should always be conducted sensitively, led by the neuromuscular consultant, who knows the patient well, and supported by a specialist nurse, care advisor and/or clinical neuropsychologist.

Close liaison with a palliative medicine consultant can really be helpful with 'symptom control' management, especially for those patients with distressing symptoms such as intractable pain, where standard treatment of secretion management has failed, withdrawal of NIV at the patient's request or end stage cardiomyopathy [124-126]. A traffic light system is useful to identify those patients that are more at risk of dying and ensure that timely discussions are initiated and if reciprocated, the discussion taken further [127]. Few adults with DMD access hospice care, although many would have accessed children's respite hospices. Children's and adult's hospices are very different organisations offering different types of care. Adult palliative care services tend to offer a managed 'episode of care' approach, including 'hospice at home' which may provide valuable support for dying patients. Close collaboration between the neuromuscular team and palliative care consultant may be hugely helpful, especially in terms symptom management in patients with more advanced disease.

Key points

- Depression, anxiety, phobias, OCD and autism are common in DMD patients

- The multi-disciplinary team should include a clinical neuropsychologist
- Anxiolytic, anti-depressant therapy and or psychological intervention should be considered when there are symptoms

- All adults with DMD should be able to access a palliative care consultant imbedded within the hospital setting for symptom control

- It is helpful to discuss an advance care plan to understand the patient's wish regarding ceiling of care and priorities.

- Sensitive discussions should always be supported by a clinical nurse specialist, care advisor and or neuropsychologist

\section{SUMMARY}

Adults with DMD have complex health needs and should be seen in centres with experience and expertise in the management of DMD. Adults with DMD are a growing and heterogeneous population comprising steroid naive, previously steroid treated and those currently on steroids (either daily or intermittent), all patients are at risk of multi-system complications many of which are potentially treatable. This guidance has been produced to augment, but not replace, the international standards of care [1-3] and other management guidelines [4-6] specifically taking into account the complex medical needs of the adult DMD patient. There are areas where natural history data and research are lacking and further research is required, the Adult North Star Database will provide data in years to come.

Adults with DMD on corticosteroids require proactive management to mitigate against the on-going complications of steroids and the need to initiate NIV is likely to be delayed in this group. There is no evidence that starting steroids in adults is beneficial, however, stopping steroids should only be considered when side-effects outweigh benefit or if the patient chooses to discontinue. All patients require regular monitoring of respiratory function and screening for nocturnal hypoventilation. Patients on steroids are also at risk of obstructive sleep apnoea. Cardiac function should be monitored at least 12 monthly and all patients should be receiving an ACEi and/or beta blocker with consideration of adding epleronone. Cardiac drug therapy must be carefully titrated and monitored and additional agents may be required. There should be regular monitoring of FBC and renal function (Cystatin C) as markers of renal insufficiency. Assessment of swallowing and nutritional 
status should be included in the regular assessment of patients. There should be good lines of communication between all specialists looking after DMD adults, with sharing of information. There should be special consideration and planning for any general anaesthetic with close communication and coordination between all of the specialists involved in the patient's care. Psychological support to improve participation and reduce symptoms of anxiety and depression should be made available to all patients, where necessary medical treatment should also be considered for depression and anxiety. Adult DMD patients approaching end of life should have access to a palliative care consultant to manage their symptoms and discuss an advance care plan.

\section{ACKNOWLEDGMENTS}

We are grateful to Muscular Dystrophy UK (MDUK) for funding this project and to Bobby Ancil from MDUK for his help in organising and co-ordinating the workshops. Professor Quinlivan is also funded by the NIHR UCLH Biomedical Research Centre. We would like to thank the ENMC for organising and funding the 236th Workshop on bone protection in DMD. We would like to thank Phillippa Farrant, Shelley Simmonds and Jon Hastie for their input into this document from a service user perspective.

\section{CONFLICT OF INTEREST}

The authors report no conflict of interest related to this work.

\section{REFERENCES}

[1] Birnkrant D, Bushby K, Bann C, et al. Diagnosis and management of Duchenne muscular dystrophy, part 1: Diagnosis, and neuromuscular, rehabilitation, endocrine, and gastrointestinal and nutritional management. Lancet Neurology. 2018;17(3):251-67.

[2] Birnkrant D, Bushby K, Bann C, et al. Diagnosis and management of Duchenne muscular dystrophy, part 2: Respiratory, cardiac, bone health and orthopaedic management. Lancet Neurology. 2018;17(4):347-36.

[3] Birnkrant D, Bushby K, Bann C, et al. Diagnosis and management of Duchenne muscular dystrophy, part 3; primary care, emergency management, psychosocial care, and transitions of care across the lifespan. Lancet Neurology. 2018;17(5):445-45.

[4] Sze Choong Wong, Volker Straub, Leanne M. Ward, Ros Quinlivan. 236th ENMC International Workshop Bone protective therapy in Duchenne muscular dystrophy:
Determining the feasibility and standards of clinical trials Hoofddorp, The Netherlands, 1-3 June 2018. Neuromuscular Disorders. 2019;29:251-9.

[5] John Bourke, Michaela Guglieri, Denis Duboc on behalf of the ENMC $238^{\text {th }}$ Study Group. $238^{\text {th }}$ International workshop: Updating management recommendations of cardiac dystrophinopathy. Hoofddorp, The Netherlands, 30 November-2 December 2018. Neuromuscular Disorders. 2019;29:634-43.

[6] Simpson H, Tomlinson J, Wass J, Dean J, Arlt W. Guidance for the prevention and emergency management of adult patients with adrenal insufficiency. Clinical Medicine. 2020;20:371-8.

[7] Mayer O, Finkel RS, Rummey C, Benton MJ, Glannzman AM, Flickinger J, Lindstrom BM, Meier T. Characterization of pulmonary function in Duchenne Muscular Dystrophy Pediatr. Pulmonol. 2015;50(5):487-94.

[8] Trucco F, Domingos J, Tay C, et al. Cardiorespiratory progression over 5 yeas and role of corticosteroids in Duchenne muscular dystrophy: A single-site retrospective longitudinal study. Chest. 2020;158:1606-16.

[9] Sawnani H, Thampratankul L, Szczesniak RD, Fenchel MC, Simakajornboon N. Sleep Disordered Breathing in Young Boys with Duchenne Muscular Dystrophy. J Paediatrics. 2015:166;640-5.

[10] Hull J, Aniapravan R, Chan E, Chatwin M, Forton J, Gallagher J, Gibson N, Gordon J, Hughes I, McCulloch R, Ross Russell R. Anita Simonds British Thoracic Society guideline for respiratory management of children with neuromuscular weakness. Thorax. 2012:67;i1-i40.

[11] Georges M, Nguyen-baranoff D, Griffon L, Foignot C, Bonniaud P. Phillipe Camus, jean-louis pepin, Claudio Rabec Usefulness of transcutaneous $\mathrm{PCO}_{2}$ to assess hypoventilation in restrictive lung disorders. Respirology. 2016;21:1300-6.

[12] Farrero E, Anton A, Egen C, et al. Guidelines for the management of respiratory complications in patients with neuromuscular disease. Arch Bronconeurol. 2013;49(7):306-13.

[13] Segura LG, Lorenz JD, Weingarten TN, et al. Anaesthesia and Duchenne or Becker muscular dystrophy: Review of 117 anaesthetic exposures. Pediatric Anaesthesia. 2013;23:855-86.

[14] Van Bruggen HW, Van Den Engel-Hoek L, Steenks MH, et al. Reduced mandibular range of motion in Duchenne Muscular Dystrophy: Predictive factors. Journal of Oral Rehabilitation. 2015;42:430-8.

[15] Botteron S, Verdebout CM, Jeannet P-Y, Kiliaridis S. Orofacial dysfunction in Duchenne muscular dystrophy. Archives of Oral Biology. 2009;54:26-31.

[16] American Academy of Pediatrics. Cardiovascular Health Supervision for Individuals Affected by Duchenne or Becker Muscular Dystrophy. Pediatrics. 2005;116;156973.

[17] Cripe LH, Tobias JD. Cardiac considerations in the operative management of the patient with Duchenne or Becker muscular dystrophy. Pediatric Anesthesia. 2013;23:77784.

[18] Almenrader N, Patel D. Spinal fusion surgery in children with non-idiopathic scoliosis: Is there a need for routine postoperative ventilation? British Journal of Anaesthesia. 2006;97(6):851-7.

[19] Marsh A, Edge G, Lehovsky J. Spinal fusion in patients with Duchenne's muscular dystrophy and a low forced vital capacity. Eur Spine J. 2003;12:507-12. 
[20] Harper CM, Ambler G, Edge G. The prognostic value of pre-operative predicted forced vital capacity in corrective spinal surgery for Duchenne's muscular dystrophy. Anaesthesia. 2004;59:1160-116.

[21] Jenkins JG, Bohn D, Edmonds JF, Levison H, Barker GA. Evaluation of pulmonary function in muscular dystrophy patients requiring spinal surgery. Crit Care Med. 1982;10:645-9.

[22] Gill I, Eagle M, Mehta JS, Gibson MJ, Bushby K, Bullock R. Correction of Neuromuscular Scoliosis in Patients With Preexisting Respiratory Failure. Spine. 2006;31:2478-83.

[23] Larach MG, Rosenberg H, Gronert GA, Allen GC. Hyperkalemic Cardiac Arrest During Anesthesia in Infants and Children with Occult Myopathies. Clinical Pediatrics. 1997;36:9-16.

[24] Hayes J, Veyckemans F, Bissonette B. Duchenne muscular dystrophy: An old anesthesia problem revisited. Pediatric Anesthesia. 200818:100-6.

[25] Schmidt J, Muensterz T, Wick S, Forst J, Schmitt HJ. Onset and duration of mivacurium-induced neuromuscular block in patients with Duchenne muscular dystrophy. British Journal of Anaesthesia. 2005;95(6):769-72.

[26] Muensterz T, Schmidt J, Wick S, Forst J, Schmitt HJ. Rocuronium 0.3mg.kg-1 (ED95) induces a normal peak effect but an altered time course of neuromuscular block in patients with Duchenne's muscular dystrophy. Pediatric Anesthesia. 2006;16:840-5.

[27] Ririe DG, Shapiro F, Sethna NF. The Response of Patients with Duchenne's Muscular Dystrophy to Neuromuscular Blockade with Vecuronium. Anesthesiology. 1998;88:351-4.

[28] De Boer HD, Van Esmond J, Booij LHJD J, Driessen JJ. Reversal of rocuronium-induced profound neuromuscular block by sugammadex in Duchenne muscular dystrophy. Pediatric Anesthesia. 2009;19:1226-8.

[29] Birnkrant DJ, Petelenz KM, Ferguson RD, Martin JE, Gordon GJ. Use of the Laryngeal Mask Airway in Patients With Severe Muscular Dystrophy Who Require Sedation or Anesthesia. Pediatric Pulmonology. 2006;41:107781.

[30] Bach JR, Gonzalez M, Sharma A, Swan K, Patel A. Open gastrostomy for noninvasive ventilation users with neuromuscular disease. Am J Phys Med Rehabil. 2010;89:1-6.

[31] Rajmala X, Savita S, Kirti K, Nandini X. Intravenous anaesthesia combined with peribulbar block in a child with suspected Duchenne muscular dystrophy. Acta Anaesthesiol Scand. 2004;48:1341.

[32] Bang SU, Kim YS, Kwon, Lee SM, Kim SH. Peripheral nerve blocks as the sole anesthetic technique in a patient with severe Duchenne muscular dystrophy. J Anesth. 2016;30:320-3.

[33] Skan EC, Sener M, Kocum A, Aribogan AS. Duchenne muscular dystrophy: How I do it? Regional or general anesthesia? Pediatric Anesthesia. 2009;19:624-5.

[34] Jaber S, Lescot T, Futier E. Effect of Noninvasive Ventilation on Tracheal Reintubation Among Patients With Hypoxemic Respiratory Failure Following Abdominal Surgery. A Randomized Clinical Trial. JAMA. 2016;315(13):1345-53.

[35] Lumbierresa M, Pratsa E, Farreroa E. Noninvasive positive pressure ventilation prevents postoperative pulmonary complications in chronic ventilators users. Respiratory Medicine. 2007;101:62-8.

[36] Laura J, Miske LJ, Hickey EM, Kolb SM, Weiner DJ, Panitch HB. Use of the Mechanical In-Exsufflator in Pedi- atric Patients With Neuromuscular Disease and Impaired Cough. Chest. 2004;125:1406-12.

[37] Bach JR, Ishikawa Y, Kim H. Prevention of Pulmonary Morbidity for Patients With Duchenne Muscular Dystrophy. Chest. 1997;112:1024-28.

[38] Doris TE, Bowron A, Armstrong A, Messer B. Ketoacidosis in Duchenne muscular dystrophy: A report on 4 cases. Neuromuscular Disorders. 2018;28(8):665-70.

[39] Kamdar F, Garry DJ. Dystrophin-Deficient Cardiomyopathy. J Am Coll Cardiol. 2016;67(21):2533-46.

[40] Matsumura T, Saito T, Fujimura H, et al. A longitudinal cause-of-death analysis of patients with Duchenne muscular dystrophy. Rinsho Shinkeigaku. 2011;51:743-50.

[41] John P. Bourke, Teofila Bueser, Rosaline Quinlivan. Interventions for preventing and treating cardiac complications in Duchenne and Becker muscular dystrophy and X-linked dilated cardiomyopathy. Cochrane library. 2018: Issue 10; CD009068.

[42] Duboc D, Meune C, Lerebours G, Devaux J-Y, Vaksmann G, Bécane H-M. Effect of perindopril on the onset and progression of left ventricular dysfunction in Duchenne muscular dystrophy. Journal of the American College of Cardiology. 2005;45(6):855-7.

[43] Duboc D, Meune C, Pierre B, Wahbi K, Eymard B, Toutain T, et al. Perindopril preventive treatment on mortality in Duchenne muscular dystrophy: 10 years follow-up. American Heart Journal. 2007;154(3):596-602.

[44] Raman S, Hor K, Mazur W, Halnon N, Kissel $\mathrm{J}$, He X, et al. Eplerenone for early cardiomyopathy in Duchenne muscular dystrophy: A randomised, double-blind, placebo-controlled trial. Lancet Neurology. 2015;14(2):153-61.

[45] Masood SA, Kazmouz S, Heydemann P, Li H, Kenny D. Under-recognition of Low Blood Pressure Readings in Patients with Duchenne Muscular Dystrophy. Pediatric Cardiology. 2015;36(7):1489-94.

[46] Llubani R, Vukadinovic D, Werner C, Marx N, Zewinger S, Bohm M. Hyperkalaemia in Heart FailurePathophysiology, Implications and Therapeutic Perspectives. Current Heart Failure Reports. 2018;15(6):390-7.

[47] Motoki T, Shimizu-Motohashi Y, Komaki H, et al. Treatable renal failure found in non-ambulatory Duchenne muscular dystrophy patients. Neuromuscular Disorders. 2015;25:754-7.

[48] Barber BJ1, Andrews JG, Lu Z, West NA, Meaney FJ, Price ET, Gray A, Sheehan DW, Pandya S, Yang M, Cunniff C. Oral corticosteroids and onset of cardiomyopathy in Duchenne muscular dystrophy. J Pediatr. 2013; 163(4):1080-4.e1. doi: 10.1016/j.jpeds.2013.05.060. Epub 2013 Jul 15.

[49] Adorisio R, Calvieri C, Cantarutti N, D'Amico A, Catteruccia M, Bertini E, et al. Heart rate reduction strategy using ivabradine in end-stage Duchenne cardiomyopathy. Int J Cardiol. 2019;280:99-103.

[50] Li JM, Chen H. Recurrent hypotension induced by sacubitril/valsartan in cardiomyopathy secondary to Duchenne muscular dystrophy: A case report. World Journal of Clinical Cases. 2019;7(23):4098-105.

[51] Zannad F, et al. SGLT2 inhibiotrs in patients with heart failure with reduced ejection fraction: A meta-analysis of the EMPEROR-Reduced and DAPA-HF trials. Lancet. 2020;396(10254):819-29.

[52] Menon SC, Etheridge SP, Liesemer KN, Williams RV, Bardsley T, Heywood MC, et al. Predictive value of myocardial delayed enhancement in Duchenne 
muscular dystrophy. Pediatric Cardiology. 2014;35(7): 1279-85.

[53] Villa CR, Czosek RJ, Ahmed H, Khoury PR, Anderson JB, Knilans TK, et al. Ambulatory Monitoring and Arrhythmic Outcomes in Pediatric and Adolescent Patients With Duchenne Muscular Dystrophy. Journal of the American Heart Association. 2015;5(1).

[54] Corrado G, Lissoni A, Beretta S, Terenghi L, Tadeo G, Foglia-Manzillo G, et al. Prognostic value of electrocardiograms, ventricular late potentials, ventricular arrhythmias, and left ventricular systolic dysfunction in patients with Duchenne muscular dystrophy. Am J Cardiol. 2002;89(7):838-41.

[55] Fayssoil A, Ogna A, Chaffaut C, Lamothe L, Ambrosi $\mathrm{X}$, Nardi $\mathrm{O}$, et al. Natural history of cardiac function in Duchenne and Becker muscular dystrophies on home mechanical ventilation. Medicine (Baltimore). 2018;97(27):e11381

[56] Chronic heart failure in adults: Management. NICE Clinical guideline [CG108] August 2010.

[57] Bardy GH, Kerry LL, Mark DB, Poole JE, Packer $\mathrm{DL}$, Boineau R, et al. Amiodarone or an Implantable Cardioverter-Defibrillator for Congestive Heart Failure. N Engl J Med. 2005;352:225-37. [SCD-HeFT]

[58] Chiang DY, Allen HD, Kim JJ, Valdes SO, Wang Y, Pignatelli RH, et al. Relation of cardiac dysfunction to rhythm in patients with Duchenne or Becker muscular dystrophy. Am J Cardiol. 2016;117:1349-54.

[59] Fayssoil A, Lazarus A, Wahbi K, Ogna A, Nardi O, Lofaso F, Clair B, Orlikowski D, Annane D. Cardiac implantable electronic devices in tracheotomized muscular dystrophy patients: Safety and risks. Int J Cardiol. 2016;222:975-7. DOI: 10.1016/j.ijcard.2016.08.040

[60] Fayssoil A, Lazarus A, Wahbi K, Ogna A, Nardi O, Lofaso F, Clair B, Orlikowski D, Annane D. Cardiac implantable electronic devices in tracheotomized muscular dystrophy patients: Safety and risks. Int J Cardiol. 2016;222:975-7. DOI: 10.1016/j.ijcard.2016.08.040

[61] Hiermeier UM, Baker C, Bourke JP. Exploring the acceptability of implantable cardioverter defibrillators in patients with cardiac dystrophinopathy and carers. Open Heart. 2020;7:e001230. doi:10.1136/ openhrt-2019-001230.

[62] Ponikowski P, Voors AA, Anker SD, et al. guideline for the diagnosis and treatment of acute and chronic heart failure: The task force for the diagnosis and treatment of acute and chronic heart failure of The European society of Cardiology (ESC). Developed with th special contribution of the Heart Failures Association (HFA) of the ESC. Eur J Heart Failure. 2016;18(8):891-975.

[63] Burnett H, Earley A, Voors AA, et al. Thirty years of evidence on the efficacy of drug treatmentsfor chronic heart failure with reduced ejection fraction. Circ Heart Fail. 2017; 10:e03529.

[64] Zannard F, alla F, Dousset B, Perez A, Pitt B. Limitation of excessive extracellular matrix turn over may contribute to survival benefit of spironolactone therapy in patients with congestive heart failure: Insights from the randomised Aldosterone evaluation study (RALES). RALES investigators, Circulation. 2001;102(22):2700-6.

[65] Cicoira M, Zanolla L, Franceschini L et al. Relation of aldosterone 'escape' despite in angiotensin converting enzyme inhibitor administration to inpaired exercise capacity in chronic congestive heart failure secondary to ischaemic or idiopathic dilated cardiomyopathy. Am J Cardiol. 2002;89(4):403-7.
[66] Zannad F, alla F, dousset B, Perez A, Pitt B. Limitation of excessive extracellular matrix turnover may contribute to survival benefit of spironolactone therapy in patients with congestive heart failure: Insights from the randomised aldosterone evaluation study (RALES). RALES investigators. Circulation. 2001;102(22):2700-6.

[67] Australia/New Zealand Heart failure Research Collaborative Group. Randomised placebo controlled trial of carvedilol in patients with congestive heart failure due to ischaemic heart disease. Lancet. 1997;349(9049): 11181-9.

[68] Takahiro Motoki, Yuko Shimizu-Motohashi, Hirofumi Komaki, Madoka Mori-Yoshimura, Yasushi Oya, Eri Takeshita, Akihiko Ishiyama, Takashi Saito, and others. Treatable renal failure found in non-ambulatory Duchenne muscular dystrophy patients. Neuromuscular Disorders. 2015;25(10):754-57.

[69] Dries DL, Exner DV, Domanski MJ, Greenberg B, Stevenson LW. The prognostic implications of renal insufficiency in asymptomatic and symptomatic patients with left ventricular systolic dysfunction. JAm Coll Cardiol. 2000;35:681-9.

[70] Braat E, Hoste L, Waele LD, et al. Renal function in children and adolescents with Duchenne muscular dystrophy. Neuromuscular Disorders. 2015;25:381-7.

[71] Villa CR, Kaddourah A, Mathew J, et al. Identifying evidence of cardio-renal syndrome in patients with Duchenne muscular dystrophy using cystatin C. Neuromuscular Disorders. 2016; 26:637-42.

[72] Kimura K, Morita H, Daimon M, et al. Utility of Cystatin C estimating glomerular filtration rate in patients with muscular dystrophy. A good agreement with insulin clearance. Int Heart J. 2016;57:386-8.

[73] Dupont M, Wu Y, Hazen SL, Tang WH. CystatinC identifies patients with stable chronic heart failure at increased risk for adverse cardiovascular events. Circ Heart Fail. 2012;5:602-9.

[74] Viollet L, Gailey S, Thornton DJ, et al. Utility of Cystatin $\mathrm{C}$ to monitor renal function in Duchenne muscular dystrophy. Muscle Nerve. 2009;40:438.

[75] Villa CR, Kaddourah A, Mathew J, et al. Identifying evidence of cardio-renal syndrome in patients with Duchenne muscular dystrophy using cystatin C. Neuromuscular Disorders. 2016;26:637-42.

[76] Silverberg DS, Wexler D, Iaina A, Schwartz D. The interaction between heart failure and other heart diseases, renal failure, and anemia. Semin Nephrol. 2006;26:296-306.

[77] Shumyatcher Y, Shah TA, Noritz GH, Brouhard BH, Spirnak JP, Birnkrant DJ. Symptomatic nephrolithiasis in prolonged survivors of Duchenne muscular dystrophy. Neuromuscul Disord. 2008;18(7):561-4.

[78] MacLeod M, Kelly R, Robb SA, et al. Bladder dysfunction in Duchenne muscular dystrophy. Arch Dis Child. 2003;88:347-9.

[79] Caress J, Kothari M, Bauer S, et al. Urinary dysfunction in Duchenne muscular dystrophy. Muscle and Nerve. 1996;19:819-22.

[80] Weatherspoon SE, Collins J, Sucharew H, Wong BL, Rybalsky I, Rose SR, Klein DJ, Rutter MM. Metformin reduces weight and BMI in Duchenne muscular dystrophy patients on long term glucocorticoid therapy. Neuromusc Disord. 2012:22:866.

[81] Ingrid E.C. Verhaart, Lenie van den Engel-Hoek, Marta L. Fiorotto, Mirjam Franken-Verbeek, Elizabeth Vroom, on behalf of the workshop participants. Nutrition in Duchenne 
muscular dystrophy 16-18 March 2018, Zaandam, the Netherlands. Neuromusc Disord. 2018;28:680-9.

[82] Arahata H, Nishiyama T, Inada K, Miyoshi A, Watanabe A, Kawano Y, Sasagasako N, Fujii N. Percutaneous endoscopic gastrostomy in patients with Duchenne muscular dystrophy compared with amyotrophic lateral sclerosis and Parkinson syndrome. Neuromusc Disord. 2017;27(Supplement 2):S102.

[83] Martigne L, Seguy D, Pellegrini N, Orlikowski D, Cuisset JM, Carpentier A, Tiffreau V, Guimber D, Gottrand F. Efficacy and tolerance of gastrostomy feeding in Duchenne muscular dystrophy. Clin Nutr. 2010;29(1):60-4.

[84] Aminda De Silva, Jeremy M D Nightingale. Refeeding syndrome: Physiological background and practical management. Frontline Gastroenterology. 2019;0:1-6.

[85] Christian M. Lo Cascio, Oliver Goetze, Tsogyal D. Latshang, Sena Bluemel, Thomas Frauenfelder, Konrad E. Bloch. Gastrointestinal Dysfunction in Patients with Duchenne Muscular Dystrophy. Plos One. 2016: October; 1-14.

[86] Kraus D, Wong BL, Horn PS, Kaul A. Constipation in Duchenne Muscular Dystrophy: Prevalence, Diagnosis, and Treatment. J Pediatr. 2016;171:183-8.

[87] Tanaka H1, Hiramatsu K, Iida T, Inoue T, Yasuoka H, Koja A, Saitoh S, Hiromatsu T. Recurrent gastric volvulus resolved surgically after therapeutic percutaneous endoscopic gastrostomy in Duchenne muscular dystrophy. Nihon Shokakibyo Gakkai Zasshi. 2012;109(3):418-24.

[88] Ricotti V, Engledow E, Emmanuel A, Knowles J, Walker D, Giordano P, Simonds A, Hanna M, et al. Recurrent pseudo-obstruction and sigmoid volvulus in Duchenne Muscular Dystrophy: A case report. Neuromusc Disord. 2012;22:887-8.

[89] Giordano P, Simonds A, Hanna M, Quinlivan R. Recurrent pseudo-obstruction and sigmoid volvulus in Duchenne Muscular Dystrophy: A case report. Neuromuscular Disorders. 2012;22:804-908.

[90] Adike A, DiBaise JK. Small intestinal bacterial overgrowth; nutritional implication, diagnosis and management. Gastroenterol. Clin North Am. 2018;47(1):193-208.

[91] David Brumbaugh, Laura Watne, Frederic Gottrand, Ann Gulyas, Ajay Kaul, Jacqueline Larson, Jean Tomezsko. Nutritional and Gastrointestinal Management of the patient with Duchenne Muscular Dystrophy. Pediatrics. 2018; Volume 142, number s2,e20180333G

[92] Tetsuhide Ito, Robert T. Jensen. Association of Long-term Proton Pump Inhibitor Therapy with Bone Fractures and effects on Absorption of Calcium, Vitamin B12, Iron, and Magnesium. Curr Gastroenterol Rep. 2010;12(6):448-57.

[93] Murphy L, Yabrodi M, Lutfi R. Fat embolism syndrome in Duchenne Muscular Dystrophy: Early recognition and aggressive therapy. Case rep Crit Care. 2018;2018:3686470.

[94] Matthew Jacques, Rachel Stockley, Emma Bostock, Jonathon Smith, Christian G DeGoede, Christopher Morse. Frequency of Reported Pain in Adult Males With Muscular Dystrophy. PLoS One. 2019;14(2):e0212437.

[95] Emma L. Bostock, Bryn T. Edwards, Matthew F. Jacques, Jake T. S. Pogson, Neil D. Reeves, Gladys L. OnambelePearson, Christopher Morse. Impaired Glucose Tolerance in Adults With Duchenne and Becker Muscular Dystrophy Nutrients. 2018;10(12):1947.

[96] Doris TE, Bowron A, Armstrong A, Messer B. Ketoacidosis in Duchenne Muscular Dystrophy: A Report on 4 Cases. Neuromuscul Disord. 2018;28(8):665-70.
[97] World Health Organization (WHO): International Classification of Functioning, Disability and Health: Children \& Youth Version. Switzerland: Geneva; 2007.

[98] Minis M, Heerkens Y, Oostendorp R, Engelen B. Classification of employment factors according to the International Classification of Functioning, Disability and Health in patients with neuromuscular disease: A systematic review. Disability \& Rehabilitation. 2009;31: 2150-63.

[99] Fowler W, Abresch R, Koch T, Brewer M, Bowden R, Wanlass R. Employment profiles in neuromuscular diseases. American Journal of Physical Medicine and Rehabilitation. 1997;76:26-37.

[100] Im S, Moon J, Park Y, Lee S. (2009). The educational and vocational status of muscular dystrophy patients. Neuromuscular Disorders.

[101] Lindsay S, Cagliostro E, McAdam L. Meaningful occupations of young adults with muscular dystrophy and other neuromuscular disorders. Canadian Journal of Occupational Therapy. 2019;86(4):277-88.

[102] Pangalila RF, van den Bos GA, Bartels B, Bergen M, Stam HJ, Roebroeck ME. Prevalence of fatigue, pain, and affective disorders in adults with Duchenne muscular dystrophy and their associations with quality of life. Archives of Physical Medicine \& Rehabilitation. 2015;96(7):1242-7, $2015 \mathrm{Jul}$.

[103] Bendixen R, Senesac C, Lott DJ, Vandenborne K. Participation and quality of life in children with Duchenne muscular dystrophy using the International Classification of Functioning, Disability and Health. Health and Quality of Life Outcomes. 2012;10(43) doi:10.1186/1477-7525$10-43$.

[104] Vandervelde L, Van den Bergh PY, Goemans N, Thonnard JL. ACTIVLIM: A Rasch-built measure of activity limitations in children and adults with neuromuscular disorders. Neuromuscul Disord. 2007;17:459-69.

[105] Abbott D, Carpenter J. 'Wasting precious time': Young men with Duchenne muscular dystrophy negotiate the transition to adulthood. Disability \& Society. 2014;29(8):1192-205.

[106] Albrecht GL, Devlieger PJ. The disability paradox: High quality of life against all odds. Soc Sci Med. 1999;48:97788.

[107] Landfeldt E, Lindgren P, Bell CF, et al. Health-related quality of life in patients with Duchenne muscular dystrophy: A multinational, cross-sectional study. Dev Med Child Neurol. 2016; 58:508-15.

[108] Pangalila RF, van den Bos GAM, Bartels B, et al. Quality of life of adult men with Duchenne muscular dystrophy in the Netherlands: Implications for car e. JRehabilMed. 2015;47:161-66.

[109] Krupp LB, LA Rocca NG, Muir-Nash J, Steinberg AD. The fatigue severity scale. Application to patients with multiple sclerosis and systemic lupus erythematosus. Arch Neurol. 1989;46(10):1121-3.

[110] Zigmond RAS, Snaith RP. The hospital anxiety and depression scale. Acta Psychiatr Scand. 1983;76(6): 361-70.

[111] Snow WM, Anderson JE, Jakobson LS. Neuropsychological and neuro-behavioural functioning in Duchenne Muscular Dystrophy: A Review. Neuroscience and Behavioural Review. 2013;37:743-52.

[112] Bushby KM, Appleton R, Anderson LV, Welch JL, Kelly P, Gardner-Medwin D. Deletion status and intellectual impairment in Duchenne muscular dystrophy. Develop- 
mental Medicine \& Child Neurology. 1995;37(3):260-9.

[113] Hinton VJ, De Vivo DC, Nereo NE, Goldstein E, Stern Y. Selective deficits in verbal working memory associated with a known genetic etiology: The neuropsychological profile of Duchenne muscular dystrophy. Journal of the International Neuropsychological Society. 2001;7:45-54.

[114] Hinton VJ, Fee RJ, Goldstein EM, De Vivo DC. Verbal and memory skills in males with Duchenne muscular dystrophy. Developmental Medicine \& Child Neurology. 2007;49:123-8.

[115] Hendriksen JGM, Vles JSH. Neuropsychiatric disorders in Males with Duchenne Muscular Dystrophy: Frequency rate of attention-deficit hyperactivity disorder (ADHD), autism spectrum disorder and obsessive-compulsive disorder. Journal of Child Neurology. 2008;23(5):477-81.

[116] Cotton S, Crowe FC, Voudouris N. Neuropsychological Profile of Duchenne Muscular Dystrophy, Child Neuropsychology: A Journal on Normal and Abnormal Development in childhood and Adolescence. 1998;4(2): 110-7.

[117] Ueda Y, Suwazano S, Maedo S, Higuchi I. Profile of cognitive function in adults with Duchenne muscular dystrophy. Brain and Development. 2017;39:225-30.

[118] Banihani R, Smile S, Yoon G, et al. Cognitive and Neurobehavioral Profile in Boys With Duchenne Muscular Dystrophy. J Child Neurol. 2015;30:1472-82.

[119] Latimer R, Street N, Conway KC, et al. Secondary Conditions Among Males With Duchenne or Becker Muscular Dystrophy. J Child Neurol. 2017;32:663-70.

[120] Pangalila RF, van den Bos GA, Bartels B, et al. Prevalence of fatigue, pain, and affective disorders in adults with Duchenne muscular dystrophy and their associations with quality of life. Arch Phys Med Rehabil. 2015;96:1242-7.
[121] Landfelt E, Edstrome J, Buccella F, Kirschner J, Lochmuller H. Duchenne muscular dystrophy and caregiver burden: A systematic review. Developmental Medicine and Child Neurology. 2018;60:987-96.

[122] Willis D, Gannon C, Jones J.' Specialist palliative care, non-cancer conditions, and guilt: An unholy triad?' International Journal of Palliative Nursing. 2014;20(3):121-3.

[123] Abbott D, Prescott H, Forbes K, Fraser J, Majumdar A. Men with Duchenne muscular dystrophy and end of life planning. Neuromuscular Disorders. 2017;27(1):38-44.

[124] Arias R, Andrews J, Pandya S, Pettit K, Trout C, Apkon S, Karwoski J, Cunniff C, Matthews D, Miller T, Davis MF, Meaney FJ. Palliative care services in families of males with Duchenne muscular dystrophy. Muscle Nerve. 2011;44(1):93-101.

[125] Hiscock A, Kuhn I, Barclay S. Advance care discussions with young people affected by life-limiting neuromuscular diseases: A systematic literature review and narrative synthesis. Neuromuscular Disorders. 2017;27(2):115-9.

[126] Rahbek J, Steffensen BF, Bushby K, de Groot IJM. 206th ENMC International Workshop: Care for a novel group of patients - adults with Duchenne muscular dystrophy Naarden, The Netherlands, 23-25 May 2014 Neuromuscular Disorders. 2015;25(9):727-38.

[127] Willis D, Vithlani R, Willis T. Using a traffic light system to identify palliative care needs in adult neuromuscular patients BMJ Supportive \& Palliative Care Mar. 2018;8(Suppl 1)A75; DOI: 10.1136/bmjspcare2018-ASP abstracts. 


\section{APPENDIX 1}

Adult North Star Network members

\begin{tabular}{|c|c|}
\hline Adam Rochester & Royal Brompton and Harefield NHS Trust \\
\hline Adnan Manzur & Great Ormond Street Hospital for Children NHS Foundation Trust \\
\hline Adrian Morley-Davies & University Hospitals of North Midlands NHS Trust \\
\hline Ajit Thomas & University Hospitals of North Midlands \\
\hline Alanna Hare & Royal Brompton and Harefield NHS Trust \\
\hline Aleksandra Pietrusz & University College London Hospitals, National Hospital for Neurology and Neurosurgery \\
\hline Alekksander Radunovic & Barts Healthcare Trust \\
\hline Alice Roe & University Hospitals Birmingham NHS Foundation Trust \\
\hline Alice Wilson & University Hospital Southampton NHS Foundation Trust \\
\hline Amanda Wallace & University college Hospitals NHS Trust \\
\hline Andria Merrison & South West Neuromuscular Operational Delivery Network \\
\hline Andy Rose & Cambridge University Hospitals NHS Foundation Trust \\
\hline Angela Reddy & Guy's and St Thomas' NHS Foundation Trust \\
\hline Anita Simonds & Royal Brompton and Harefield NHS Trust \\
\hline Ann Morgan & South West Neuromuscular Operational Delivery Network \\
\hline Anna Mayhew & John Walton Muscular Dystrophy Research Centre, Newcastle \\
\hline Anna Walker & MD Support Centre \\
\hline Anton Emmanuel & University College London Hospitals, National Hospital for Neurology and Neurosurgery \\
\hline Antonis Pantazis & Royal Brompton and Harefield NHS Trust \\
\hline Ben Messer & Newcastle upon Tyne Hospitals NHS Foundation Trust \\
\hline Bobby Ancil & Muscular Dystrophy UK \\
\hline Bobby Ancil & Patient advocate, Muscular Dystrophy UK \\
\hline Caroline Hutchings & University Hospitals Southampton NHS Foundation Trust \\
\hline Channa Hewamadduma & Sheffield Teaching Hospitals Foundation NHS Trust \\
\hline Charlotte Brierley & Cambridge University Hospitals NHS fFoundation Trust \\
\hline Charlotte F Dougan & The Walton Centre NHS Foundation Trust \\
\hline Charlotte Massey & University College London Hospitals, National Hospital for Neurology and Neurosurgery \\
\hline Chiara Marini-Bettolo & John Walton Muscular Dystrophy Research Centre, Newcastle \\
\hline Chris McDermott & Sheffield Teaching Hospitals Foundation NHS Trust \\
\hline David Shakespeare & Lancashire Teaching Hospitals NHS Foundation Trust \\
\hline Derek Willis & Shrewsbury and Telford NHS Trust \\
\hline Derek Willis & Shrewsbury and Telford Hospital NHS Trust \\
\hline Dipa Jayaseelan & University College London Hospitals, National Hospital for Neurology and Neurosurgery \\
\hline Dispansu Ghosh & Leeds Teaching Hospitals NHS Trust \\
\hline Eleanor Marsh & Cardiff and Vale University Health Board \\
\hline Emily Ballard & Guy's and St Thomas' NHS Foundation Trust \\
\hline Emma Gallagher & University Hospitals Birmingham NHS Foundation Trust \\
\hline Emma Husbands & Gloucestershire Hospitals Foundation Trust \\
\hline Emma Manchester & Bradford Teaching Hospitals NHS Foundation Trust \\
\hline Emma Matthews & St George's University Hospitals foundation Trust \\
\hline Fiona Norwood & King's College Hospital NHS Foundation Trust \\
\hline Fionnuala Crummy & University College Hospitals foundation Trust \\
\hline Georgina Burke & University Hospitals Southampton NHS Foundation Trust \\
\hline Geraldine Bailey & John Walton Muscular Dystrophy Research Centre, Newcastle \\
\hline Girija Sadalage & University Hospitals Birmingham NHS Foundation Trust \\
\hline Gita Ramdharry & University College London Hospitals, National Hospital for Neurology and Neurosurgery \\
\hline Helen Chase & University Hospitals Birmingham NHS Foundation Trust \\
\hline James Lilleker & Salford Royal Teaching Hospital NHS Foundation Trust \\
\hline Jane Freebody & Oxford University Hospitals NHS Foundation Trust \\
\hline Janet McCay & South West Neuromuscular Operational Delivery Network \\
\hline Jarod Wong & University of Glasgow \\
\hline Jatin Pattni & University College London Hospitals, National Hospital for Neurology and Neurosurgery \\
\hline Javid Khan & University College London Hospitals, National Hospital for Neurology and Neurosurgery \\
\hline Jennifer Spillane & Guy's and St Thomas' NHS Foundation Trust \\
\hline Jill Davies & Cardiff and Vale University Health Board \\
\hline Jo Reffin & King's College Hospital NHS Foundation Trust \\
\hline Jodi Allen & University College London Hospitals, National Hospital for Neurology and Neurosurgery \\
\hline John Bourke & Freeman Hospital, University Hospitals, Newcastle \\
\hline Jon Hastie & Patient representative, Pathfinders UK \\
\hline
\end{tabular}




\begin{tabular}{|c|c|}
\hline Joni Cox & Norfolk Community Health and Care NHS Trust \\
\hline Jonny Smith & The Neuromuscular Centre, Winsford \\
\hline Judith Bubbear & Royal National Orthopaedic Hospital NHS Trust \\
\hline Julie Cassell & University Hopsitals of Derby and Burton \\
\hline Kate McGlashan & Colman Centre for Specialist Rehabilitation, Norwich \\
\hline Kate Russell & University College London Hospitals, National Hospital for Neurology and Neurosurgery \\
\hline Kathryn Docherty & University Hospitals Southampton NHS Foundation Trust \\
\hline Katie Nevin & Sheffield Teaching Hospitals Foundation NHS Trust \\
\hline Kirstie Spencer & Nottingham University Hospitals \\
\hline Konstantinos Koutroutsos & Sussex Kidney Unit, Brighton and Sussex University Hospitals \\
\hline Konstantinos Savvatis & National Hospital for Neurology and Neurosurgery \\
\hline Konstantinos Savvatis & Barts Health Care Trust \\
\hline Lindsay Maidment & Sheffield Teaching Hospitals Foundation NHS Trust \\
\hline Liz Househam & University Hospitals Plymouth \\
\hline Lynne Williams & Royal Papworth NHS Foundation Trust \\
\hline Mahalekshmi Desikan & National Hospital for Neurology and Neurosurgery \\
\hline Margaret Phillips & University Hopsitals of Derby and Burton \\
\hline Maria Farrugia & Scottish Muscle Network \\
\hline Maria Patasin & National Hospital for Neurology and Neurosurgery \\
\hline Marina Di Marco & Scottish Muscle Network \\
\hline Mark Busby & Bradford Teaching Hospitals NHS Foundation Trust \\
\hline Mark Roberts & Salford Royal Teaching Hospital NHS Foundation Trust \\
\hline Mark Rogers & Cardiff University Hospitals Trust \\
\hline Meredith James & John Walton Muscular Dystrophy Research Centre, Newcastle \\
\hline Michael Davies & Royal Papworth NHS Foundation Trust \\
\hline Michelle Ennis & The Walton Centre NHS Foundation Trust \\
\hline Nicholas Emery & The Robert Jones and Agnes Hunt Orthopaedic Hospital \\
\hline Nick Davies & University Hospitals Birmingham NHS Foundation Trust \\
\hline Nick Hart & Guy’s \& St Thomas' NHS Foundation \\
\hline Nicola Grose & South West Neuromuscular Operational Delivery Network \\
\hline Nicola White & The Walton Centre NHS Foundation Trust \\
\hline Patrick Murphy & Guy's and St Thomas' NHS Foundation Trust \\
\hline Paul Orme & The Neuromuscular Centre, Winsford \\
\hline Paula Fenty & Nottingham University Hospitals NHS Foundation Trust \\
\hline Phil Kelly & Salford Royal Teaching Hospital NHS Foundation Trust \\
\hline Phillipa Farrant & Patient representative, Duchenne support Group \\
\hline Priya Shnamugarjah & York Teaching Hospital NHS Foundation Trust \\
\hline Rachel Ibbotson & Derby Neuromuscular Service \\
\hline Rahul Mukhergee & Birmingham Heartlands Hospital \\
\hline Rebecca Flesher & The Walton Centre NHS Foundation Trust \\
\hline Richa Kulshrestha & The Robert Jones and Agnes Hunt Orthopaedic Hospital \\
\hline Richard Keen & Royal National Orthopaedic Hospital NHS Trust \\
\hline Ronan Astin & University College London Hospitals, National Hospital for Neurology and Neurosurgery \\
\hline Ros Quinlivan & University College London Hospitals, National Hospital for Neurology and Neurosurgery \\
\hline Saam Sedehizadeh & Nottingham University Hospitals NHS Foundation Trust \\
\hline Sally Glover & University Hospitals Birmingham NHS Foundation Trust \\
\hline Samantha Wood & Lancashire \& South Cumbria NHS Foundation Trust \\
\hline Sarah Gates & Morriston Hospital Swansea \\
\hline Sarah Holmes & University College London Hospitals, National Hospital for Neurology and Neurosurgery \\
\hline Sarah Mason & Belfast City Hospital \\
\hline Shagufay Mahendran & The Walton Centre NHS Foundation Trust \\
\hline Shelley Simmonds & Patient representative, Action Duchenne \\
\hline Shelley Srivastava & Guy’s \& St Thomas' NHS Foundation \\
\hline Sherryl Chatfield & University College Hospitals Foundation Trust \\
\hline Simon Baudoin & Newcastle upon Tyne Hospitals NHS Foundation Trust \\
\hline Siobhan Macauley & Belfast City Hospital \\
\hline Stam Kapetanakis & Guy's \& St Thomas' NHS Foundation \\
\hline Stefan Brady & Oxford University Hospitals NHS Foundation Trust \\
\hline Sunitha Narayan & University Hospital Southampton NHS Foundation Trust \\
\hline Tim Quinnell & Royal Papworth NHS Foundation Trust \\
\hline Tracey Adjei & Hull and East Yorkshire Hospitals NHS Trust \\
\hline Tracey Willis & The Robert Jones and Agnes Hunt Orthopaedic Hospital \\
\hline Venkataramanan Srinivasan & University Hospitals Birmingham NHS Foundation Trust \\
\hline Yvonne Julien & University Hospitals of Leicester \\
\hline
\end{tabular}

